\title{
A variational approach to chaotic dynamics in periodically forced nonlinear oscillators *
}

by

\author{
Elena BOSETTO, Enrico SERRA ${ }^{1}$ \\ Dipartimento di Matematica, Politecnico di Torino, Corso Duca degli Abruzzi, 24, \\ 10129 Torino, Italy
}

Manuscript received 9 January 1999, revised 26 April 2000

ABSTRACT. - We prove that a class of problems containing the classical periodically forced pendulum equation displays the main features of chaotic dynamics. The approach is based on the construction of multibump type heteroclinic solutions to periodic orbits by the use of global variational methods.

(C) 2000 L'Association Publications de l'Institut Henri Poincaré. Published by Elsevier B.V. All rights reserved

RÉSUMÉ. - On prouve qu'une classe de problèmes contenant l'équation du pendule forcé périodiquement possède les principales caractéristiques de la dynamique chaotique. Les techniques sont basées sur la construction d'orbites hétéroclines de type multibump asymptotiques à des solutions périodiques. Les methodes sont variationnelles globales. (C) 2000 L'Association Publications de l'Institut Henri Poincaré. Published by Elsevier B.V. All rights reserved

\footnotetext{
* Work supported by MURST, Project "Metodi variazionali ed equazioni differenziali non lineari".

${ }^{1}$ E-mail: serra@polito.it.
} 


\section{INTRODUCTION}

The purpose of this paper is to produce evidence of chaotic dynamics in the equation

$$
\ddot{u}(t)+W^{\prime}(t, u(t))=h(t)
$$

through the existence of heteroclinic type solutions. In (1), $W^{\prime}$ stands for $\frac{\partial W}{\partial u}$, and the functions $W$ and $h$ satisfy the following assumptions:

(H1) $W \in \mathcal{C}^{2}(\mathbf{R} \times \mathbf{R} ; \mathbf{R})$ is 1-periodic in $t$ and $S$-periodic in $u$,

(H2) $h \in \mathcal{C}(\mathbf{R} ; \mathbf{R})$ is 1-periodic and satisfies $\int_{0}^{1} h(t) d t=0$.

Assumptions ( $\mathrm{H} 1)$ and $(\mathrm{H} 2)$, which we will use throughout the paper without further repetition, are satisfied by the periodically forced pendulum equation $\ddot{u}+\sin u=h(t)$, where $h$ has mean value zero. We believe that the applicability of our results to this classical mechanical model is one of the points of interest of the present work.

In order to describe our results, let $V(t, u)=W(t, u)-h(t) u$ and let $L(u)=\frac{1}{2} \dot{u}^{2}-V(t, u)$ be the Lagrangian associated to (1). It is well known that equation (1) admits, under assumptions (H1) and (H2), an ordered family of 1-periodic solutions which can be obtained as global minimizers of the action functional

$$
f(u)=\int_{0}^{1} L(u) d t
$$

over the space $E_{1}$ of 1-periodic, $H_{l o c}^{1}$ functions. Let $u_{0}$ and $u_{1}$ be two consecutive minimizers (see Definition 2.1). We are interested in solutions $q$ of (1) which are asymptotic to prescribed states in $\left\{u_{0}, u_{1}\right\}$ when $t$ tends to $\pm \infty$. To simplify notation, we will write $q(-\infty)=$ $u_{0}$ instead of $\lim _{t \rightarrow-\infty}\left(q(t)-u_{0}(t)\right)=0$ for example, and similar expressions at $+\infty$. A solution is called homoclinic if it is asymptotic to the same state at $-\infty$ and $+\infty$, and heteroclinic if the asymptotic states are different.

The search for homoclinic or heteroclinic solutions is a classical subject, which has been deeply studied by the use of geometrical methods (see, e.g., [23]); in this context, the results are generally of a perturbative nature. Starting from an integrable system, one analyzes the dynamics of a new system obtained as a small perturbation of the former.

A second class of methods, global in nature, is provided by the AubryMather theory of monotone twist maps. This is a rich and well developed 
theory that deals with discrete dynamical systems and that has more than one point in common with our approach. We refer the reader to [12] and to the detailed references therein and, as an introduction to the theory, to the paper [4].

More recently, a global approach based on purely variational arguments has been shown to be useful in proving existence and multiplicity of homoclinic solutions for certain classes of problems. In what follows, we reserve the term "variational approach" to the latter class of methods, although also the Aubry-Mather theory makes use of variational type arguments.

The use of the variational approach is often convenient because it does not require the system to be a small perturbation of a simpler one, needs in general only mild nondegeneracy conditions, and is powerful enough to detect the principal features of chaotic dynamics. This approach has been very extensively applied, starting from the late eighties, to problems of Duffing kind with various types of time dependence (see $[5,9,10,14$, $19,20,22]$, and references therein for an introduction to the subject). The object of these papers is to produce evidence of chaotic dynamics by the construction of multibump type homoclinic orbits. In this type of problem the solutions are homoclinic to a rest point of the system.

A rather striking characteristic of the variational approach is that only a few papers are devoted to the study of homoclinic or heteroclinic solutions to periodic orbits, a reference model in this case being the forced pendulum equation. This is possibly due to the lack of a simple functional formulation for such problems, which, by their very nature, force one to work with nonintegrable functions. The major contribution to the removal of this obstacle is the work [16] by P.H. Rabinowitz, where the author managed to construct a functional $J$ (see (2)) whose minimizers are the desired solutions, opening in this way the road to a global approach. In the papers [17,18,13,8,1], which followed [16], existence and multiplicity of heteroclinics, homoclinics and more general multibump and chaotic solutions were obtained both for scalar problems and for systems. The common feature of all these papers, and the idea that led Rabinowitz to construct the functional $J$, is the use of a reversibility assumption: it is required that the potential $V$ be an even function of time.

The purpose of our work is to show that for scalar problems one can drop the reversibility assumption and obtain the same type of results as in the reversible case.

The main difficulty one has to face when working without reversibility assumptions is to show that the functional $J$ still makes sense and is fit 
to obtain the desired results. After the construction of the functional, the typical way to prove existence of multibump and chaotic trajectories can be roughly synthesized in the following scheme.

First, one usually needs some nondegeneracy assumption on the asymptotic states $u_{0}$ and $u_{1}$. This means for example that $u_{0}$ and $u_{1}$ are required to be nondegenerate as minimizers, or at least isolated; these assumptions have been used in [15] and [16-18] respectively. Then, the first basic types of heteroclinic solutions can be found; in our problem these are an orbit $q_{0}$ that connects $u_{0}$ to $u_{1}$ (namely $q_{0}(-\infty)=u_{0}$ and $\left.q_{0}(+\infty)=u_{1}\right)$ and an orbit $q_{1}$ that connects $u_{1}$ to $u_{0}$. The basic nature of $q_{0}$ and $q_{1}$ is their appearance as minimizers of the functional $J$ over suitable classes of functions. These orbits are then used as building blocks for more complicated types of solutions, in the spirit of the shadowing lemma; they give rise to multibump type orbits, which are solutions that oscillate between the states $u_{0}$ and $u_{1}$ a prescribed number of times and are asymptotic to the required states at $\pm \infty$. In order to do this, one needs a further nondegeneracy condition on the structure of the basic heteroclinic solutions. This assumption takes the place of the transversality condition in the perturbative approach and prevents, for example, the problem from being autonomous. Finally, a rather careful analysis is needed in order to obtain estimates independent of the number of bumps, so that one can pass to the limit and obtain solutions with infinitely many bumps, which display the chaotic nature of the dynamics.

With respect to this general scheme our work is also characterized, in addition to the removal of the reversibility assumption, by the following features.

The nondegeneracy conditions on $u_{0}$ and $u_{1}$ are replaced with the requirement that $u_{0}$ and $u_{1}$ be consecutive. We work throughout the paper in the order interval $\left[u_{0}, u_{1}\right]$; this weakens the classical assumptions and shows that our results are in general valid for variational problems possessing two global minimizers, without assumptions on the space periodicity of the potential. On the other hand, the existence of consecutive minimizers is necessary in order to obtain multibump solutions (see [8]).

Next, the nondegeneracy condition on the structure of basic heteroclinics is a suitable modification of the one introduced in [8] for the scalar reversible case (and later adapted in [18] to reversible systems). Roughly it says that the sets of orbits connecting $u_{0}$ to $u_{1}$ and $u_{1}$ to $u_{0}$ must be disconnected (see $(*)$ at the beginning of Section 4 ). This assumption is then compared to the standard nondegeneracy hypotheses in the perturbative approach. We prove that when $u_{0}$ and $u_{1}$ are hyperbolic (which we never 
require), our condition is equivalent to the fact that the stable manifold of $u_{0}$ (respectively $u_{1}$ ) and the unstable manifold of $u_{1}$ (respectively $u_{0}$ ) do not coincide.

Below we state our main results; in their statements, when we label $u$ with an index, that index must be read mod 2 .

THEOREM 1.1 (Multibump solutions). - Assume $u_{0}$ and $u_{1}$ are consecutive minimizers and let $(*)$ hold. Then for every $\delta>0$ small enough there exists $m=m(\delta) \in \mathbf{N}$ such that for every sequence $\left(p_{i}\right)_{i \in \mathbf{Z}} \subset$ $\mathbf{Z}$ such that $p_{i+1}-p_{i} \geqslant 4 m$ and for every $j, k \in \mathbf{Z}$ with $j<k$, there exists a classical solution $q$ of (1) satisfying

$$
\begin{aligned}
& u_{0}(t)<q(t)<u_{1}(t) \quad \text { for all } t \in \mathbf{R}, \\
& q(-\infty)=u_{j}, \quad q(+\infty)=u_{k+1},
\end{aligned}
$$

and, for all $i=j, \ldots, k$,

$$
\left|q\left(p_{i}-m\right)-u_{i}\left(p_{i}-m\right)\right| \leqslant \delta \quad \text { and } \quad\left|q\left(p_{i}+m\right)-u_{i+1}\left(p_{i}+m\right)\right| \leqslant \delta \text {. }
$$

THEOREM 1.2 ( $\infty$-bump solutions). - Under the same assumptions as in Theorem 1.1, for every $\delta>0$ small enough there exists $m=m(\delta) \in$ $\mathbf{N}$ such that for every sequence $\left(p_{i}\right)_{i \in \mathbf{Z}} \subset \mathbf{Z}$ such that $p_{i+1}-p_{i} \geqslant 4 m$, there exists a classical solution $q$ of (1) satisfying

$$
u_{0}(t)<q(t)<u_{1}(t) \quad \text { for all } t \in \mathbf{R},
$$

and

$$
\left|q\left(p_{i}-m\right)-u_{i}\left(p_{i}-m\right)\right| \leqslant \delta \quad \text { and } \quad\left|q\left(p_{i}+m\right)-u_{i+1}\left(p_{i}+m\right)\right| \leqslant \delta
$$

for all $i \in \mathbf{Z}$.

In addition to these results, one could also work in a space of periodic functions to obtain the existence of infinitely many periodic orbits with large periods. This requires only minor modifications of our arguments, and we will not deepen this point; we refer the reader to [11] for a similar construction in a different setting.

The above results show that some of the principal features of chaotic dynamics are embodied in Eq. (1); that is, one has sensitive dependence from the initial conditions, existence of infinitely many periodic orbits 
with diverging periods, and existence of an uncountable number of bounded, nonperiodic trajectories.

The nature of our methods also shows that the set of forcing terms $h$ for which (1) displays the described features is open in the space of continuous periodic functions with mean value zero.

As final remark, we point out that through the multibump structure obtained by Theorem 1.1 one can easily show the Poincaré map associated to (1) has positive topological entropy.

The main drawback of our method is that it relies deeply on the order structure of $\mathbf{R}$, so that for the time being, it does not seem to extend to systems. Some problems of existence of heteroclinics to periodic motions for systems in the nonreversible (nonperturbative) case can be however solved with the methods of [12].

This paper is structured as follows: Section 2 contains the estimates needed for the definition and the use of the functional $J$. In Section 3, we prove the existence of the basic heteroclinic solutions. The multibump construction and the main results are given in Section 4. Finally, the comparison with the classical results in the hyperbolic case is the object of Section 5.

Notation. We use the symbols $L^{p}$ and $H^{1}$ to denote the spaces $L^{p}(0,1)$ and $H^{1}(0,1)$, endowed with their usual norms. The symbols $L_{l o c}^{\infty}$ and $H_{l o c}^{1}$ stand for $L_{l o c}^{\infty}(\mathbf{R} ; \mathbf{R})$ and $H_{l o c}^{1}(\mathbf{R} ; \mathbf{R})$.

If $u_{0}$ and $u_{1}$ are 1-periodic (continuous) functions such that $u_{0}(t) \leqslant$ $u_{1}(t)$ for all $t$, we say that $u \in\left[u_{0}, u_{1}\right]$ if

$$
u_{0}(t) \leqslant u(t) \leqslant u_{1}(t) \quad \forall t \in \operatorname{dom}(u) .
$$

Whenever $E$ is a set of continuous functions we will write $u \in E \cap$ $\left[u_{0}, u_{1}\right]$ to mean that $u \in E$ and $u_{0}(t) \leqslant u(t) \leqslant u_{1}(t) \forall t \in \operatorname{dom}(u)$.

\section{FUNCTIONAL SETTING AND ESTIMATES}

This section is devoted to the study of the the main properties of the functional that we will use to construct homoclinic solutions. These properties require a number of estimates on the action of both periodic and nonperiodic functions.

To begin with we define, for $n \in \mathbf{N}$, the space of $n$-periodic functions

$$
E_{n}=\left\{u \in H_{l o c}^{1}(\mathbf{R} ; \mathbf{R}) / u(t+n)=u(t) \text { a.e. }\right\} \text {, }
$$


the Lagrangian $L(u)=\frac{1}{2} \dot{u}^{2}-V(t, u)$, the action functional over $H^{1}(0,1)$

$$
f(u)=\int_{0}^{1} L(u) d t
$$

and we set

$$
c_{p}=\min _{E_{1}} f(u) .
$$

By classical arguments, the value $c_{p}$ is attained at an ordered family of functions. It is a family because of the spatial periodicity of the potential $W$, and it is ordered since two absolute minimizers cannot cross (see [21]).

DEFINITION 2.1. - We say that $u_{0}, u_{1} \in E_{1}$ are consecutive minimizers of the periodic problem associated to $E q$. (1) if

(1) $f\left(u_{0}\right)=f\left(u_{1}\right)=c_{p}$,

(2) $u_{0}(t)<u_{1}(t)$ for all $t \in[0,1]$,

(3) $u \in E_{1} \cap\left[u_{0}, u_{1}\right]$ and $f(u)=c_{p}$ imply $u \in\left\{u_{0}, u_{1}\right\}$.

Note that $u_{0}$ and $u_{1}$ need not be isolated in $E_{1}$ : there might be other minimizers accumulating to $u_{0}$ from below or to $u_{1}$ from above. The existence of consecutive minimizers is thus a weaker condition than the isolatedness of minimizers and actually in [8] it is shown that this condition is necessary for the existence of multibump solutions. From now on we will always assume that two consecutive minimizers $u_{0}$ and $u_{1}$ are given.

We now recall the definition of the functional $J$. For $k \in \mathbf{Z}$ and $q \in H_{l o c}^{1}(\mathbf{R} ; \mathbf{R}) \cap\left[u_{0}, u_{1}\right]$ we set

$$
a_{k}(q)=\int_{k}^{k+1} L(q) d t-c_{p}
$$

and we define (formally, for the moment) a functional $J$ as

$$
J(q)=\sum_{k \in \mathbf{Z}} a_{k}(q)
$$

This functional has been introduced by P.H. Rabinowitz in [16] in the context of time-reversible equations, and has been later used in $[17,18,8,1]$ to deal again with reversible problems. The reversibility 
assumption implies that each $a_{k}(q)$ is nonnegative, so that the series is well defined (convergent or divergent to $+\infty$ ), see [16]. The functional $J$ has been used in the quoted papers to prove existence of multibump type solutions, both for scalar problems and for systems. The argument used is minimization over suitable classes of functions.

In the present work there is no reversibility assumption, and this means that the terms $a_{k}(q)$ can be negative. It is therefore not clear a priori if $J$ is bounded from below or even well defined (the series might in principle be undetermined). One of the purposes of this paper is to show that for scalar problems the functional $J$ is still the right tool to use to prove existence of multibump and chaotic trajectories.

In order to carry out our program we need a series of estimates, which we now describe. We first recall the following equality.

LEMMA 2.2. - For every $n \in \mathbf{N}$,

$$
\min _{u \in E_{n}} \int_{0}^{n} L(u) d t=n c_{p}
$$

and the minimum is attained at 1-periodic functions (the minimizers of $f$ over $\left.E_{1}\right)$.

Proof. - Equality (3) is perhaps well-known. Since we don't know a precise reference we outline here a simple proof. Let $u$ be a minimizer over $E_{n}$ (the existence of such function is straightforward); then, since the problem is 1-periodic, the function $v(t)=u(t+1)$ is a minimizer as well. But since $u$ and $v$ have the same mean value and they cannot cross (by regularity arguments and uniqueness in the Cauchy problem), it must be $u(t+1)=u(t)$ for all $t$, which proves the statement.

This result shows in particular that if $u \in E_{n} \cap\left[u_{0}, u_{1}\right]$ satisfies $\int_{0}^{n} L(u) d t=n c_{p}$, then $u \in\left\{u_{0}, u_{1}\right\}$, since $u_{0}$ and $u_{1}$ are consecutive.

In many parts of this paper we will need a way to measure the distance of a function from a set of periodic minimizers. For computational reasons we will use the notion of distance described in the following definition.

Definition 2.3. - We denote by $\mathcal{M}$ the (closed) set of 1-periodic minimizers of $f$ and by $\mathcal{U}$ the set $\left\{u_{0}, u_{1}\right\}$. We also set

$$
\mathcal{M}(t)=\{u(t) / u \in \mathcal{M}\} \quad \text { and } \quad \mathcal{U}(t)=\{u(t) / u \in \mathcal{U}\} .
$$


If $v$ is a continuous function defined on a compact set I we define as its distance from $\mathcal{M}$ the number

$$
\hat{d}(v, \mathcal{M})=\max _{t \in I} \min _{u \in \mathcal{M}}|v(t)-u(t)|,
$$

and likewise for its distance from $\mathcal{U}$.

Remark 2.4. -

(i) Notice that it may result $\hat{d}(v, \mathcal{M})=0$ and $v \notin \mathcal{M}$; this happens if $v$ lies in a continuum of minimizers.

(ii) One of the reasons for measuring in this way the distance is the following immediate consequence of the definition: if $\hat{d}(v, \mathcal{U}) \geqslant \delta$, there is a point $t^{*}$ where both $\left|v\left(t^{*}\right)-u_{0}\left(t^{*}\right)\right| \geqslant \delta$ and $\mid v\left(t^{*}\right)-$ $u_{1}\left(t^{*}\right) \mid \geqslant \delta$.

The next proposition defines a constant that we will use repeatedly.

PROPOSITION 2.5. - For every $\delta>0$ there exists $\rho(\delta)>0$ such that for all $n \in \mathbf{N}$

$$
\inf \left\{\int_{0}^{n} L(v) d t-n c_{p} / v \in E_{n}, \hat{d}(v, \mathcal{M}) \geqslant \delta\right\} \geqslant \rho(\delta) .
$$

Moreover, if $v \in E_{n}$ satisfies

$$
\operatorname{dist}(v(k), \mathcal{M}(k)) \geqslant \delta \quad \text { for all } k=1, \ldots, n-1,
$$

then

$$
\int_{0}^{n} L(v) d t-n c_{p} \geqslant n \rho(\delta) .
$$

Proof. - Call $\rho(\delta, n)$ the left-hand-side of (5). Then $\rho(\delta, n)>0$ for all $n \in \mathbf{N}$ as one easily checks by standard compactness properties and the application of Lemma 2.2 (see [16] for a similar argument). We now show that $\rho(\delta, n) \geqslant \rho(\delta, 1)$, so that (5) holds with $\rho(\delta):=\rho(\delta, 1)$ uniformly in $n$.

We proceed by induction on $n$. If $n=1$ the inequality is trivial. We assume therefore that $\rho(\delta, n-1) \geqslant \rho(\delta, 1)$ and we show that $\rho(\delta, n) \geqslant \rho(\delta, 1)$. To see this, let $v \in E_{n}$ verify $\hat{d}(v, \mathcal{M}) \geqslant \delta$ and define $g:[0, n-1] \rightarrow \mathbf{R}$ as $g(t)=v(t+1)-v(t)$. The function $g$ is continuous and vanishes at some $\tau \in[0, n-1]$, for otherwise we would have 
$v(k+1)>v(k)$ (or the reversed inequality) for all $k=0, \ldots, n-1$, which contradicts the periodicity of $v$. We have therefore a point $\tau$ where $v(\tau)=v(\tau+1)$. It is not restrictive (by shifting time, if necessary) to assume that $\tau \in(0, n-1)$. Let $v_{1}$ be the 1-periodic extension of $v_{\mid[\tau, \tau+1]}$ and let

$$
v_{n-1}(t)= \begin{cases}v(t) & \text { if } t \in[0, \tau], \\ v(t+1) & \text { if } t \in[\tau, n-1] ;\end{cases}
$$

then $v_{1} \in E_{1}, v_{n-1} \in E_{n-1}$ and at least one between $\hat{d}\left(v_{1}, \mathcal{M}\right) \geqslant \delta$ and $\hat{d}\left(v_{n-1}, \mathcal{M}\right) \geqslant \delta$ is true. Noticing that

$$
\int_{0}^{n} L(v) d t=\int_{0}^{n-1} L\left(v_{n-1}\right) d t+\int_{n-1}^{n} L\left(v_{1}\right) d t,
$$

we see that by the inductive assumption,

$$
\int_{0}^{n} L(v) d t \geqslant(n-1) c_{p}+\rho(\delta, 1)+c_{p}=n c_{p}+\rho(\delta, 1) .
$$

Since this inequality holds for all $v \in E_{n}$ such that $\hat{d}(v, \mathcal{M}) \geqslant \delta$, we have that $\rho(\delta, n) \geqslant \rho(\delta, 1)$.

To prove the second part we again proceed by induction. If $n=1$ there is nothing to prove; we therefore assume that (7) is true for $n-1$ and we prove it for $n$. To do this take $v$ in $E_{n}$ satisfying (6) and repeat the above argument to construct $v_{1}$ and $v_{n-1}$. Notice now that when $\operatorname{dist}(v(k), \mathcal{M}(k)) \geqslant \delta$ for all $k=1, \ldots, n-1$, then both $v_{1}$ and $v_{n-1}$ satisfy (6) in $E_{1}$ and $E_{n-1}$, respectively. Therefore, by the inductive assumption,

$$
\begin{aligned}
\int_{0}^{n} L(v) d t & =\int_{0}^{n-1} L\left(v_{n-1}\right) d t+\int_{n-1}^{n} L\left(v_{1}\right) d t \\
& \geqslant(n-1) c_{p}+(n-1) \rho(\delta)+c_{p}+\rho(\delta),
\end{aligned}
$$

and the proof is complete.

We now turn to estimates on nonperiodic functions. The one provided by the following proposition will play a central role in the rest of the paper. Before stating it we observe that Proposition 2.5 holds also for $\delta=0$. In this case, of course, we obtain $\rho(0)=0$. 
Proposition 2.6. - There exists a positive constant $\hat{C}$ such that for every $\delta \geqslant 0$ and for every $n \in \mathbf{N}$, if $q \in H^{1}(0, n) \cap\left[u_{0}, u_{1}\right]$ satisfies $\hat{d}(q, \mathcal{U}) \geqslant \delta$, then

$$
\int_{0}^{n} L(q) d t-n c_{p} \geqslant \rho(\delta)-\hat{C}|q(n)-q(0)| .
$$

Proof. - If $\hat{d}(q, \mathcal{U}) \geqslant \delta$, there is a point $t^{*} \in[0, n]$ where $u_{1}\left(t^{*}\right)-$ $q\left(t^{*}\right) \geqslant \delta$ and $q\left(t^{*}\right)-u_{0}\left(t^{*}\right) \geqslant \delta$. We assume that $t^{*} \in\left[\frac{n}{2}, n\right]$, the other case being symmetric.

Define $v:\left[0, \frac{1}{2}\right] \rightarrow \mathbf{R}$ as $v(t)=(1-2 t)(q(n)-q(0))$ and set

$$
\varphi(t)= \begin{cases}q(t)+v(t) & \text { if } t \in\left[0, \frac{1}{2}\right] \\ q(t) & \text { if } t \in\left[\frac{1}{2}, n\right]\end{cases}
$$

then $\varphi \in E_{n}$, because $\varphi(0)=\varphi(n)$. Notice that

$$
\hat{d}(\varphi, \mathcal{M}) \geqslant \min _{u \in \mathcal{M}}\left|\varphi\left(t^{*}\right)-u\left(t^{*}\right)\right| \geqslant \delta
$$

since $u_{0}\left(t^{*}\right)<\varphi\left(t^{*}\right)<u_{1}\left(t^{*}\right)$. Applying Proposition 2.5 to $\varphi$ yields

$$
\int_{0}^{n} L(\varphi) d t-n c_{p} \geqslant \rho(\delta) .
$$

We now evaluate

$$
\begin{aligned}
\int_{0}^{n} L(\varphi) d t= & \int_{0}^{1 / 2} L(q+v) d t+\int_{1 / 2}^{n} L(q) d t \\
= & \frac{1}{2} \int_{0}^{1 / 2}\left[\dot{q}^{2}+\dot{v}^{2}+2 \dot{q} \dot{v}\right] d t-\int_{0}^{1 / 2}[V(t, q+v)-V(t, q)] d t \\
& -\int_{0}^{1 / 2} V(t, q) d t+\int_{1 / 2}^{n} L(q) d t \\
\leqslant & \int_{0}^{n} L(q) d t+M \int_{0}^{1 / 2}|v| d t+\frac{1}{2} \int_{0}^{1 / 2} \dot{v}^{2} d t+\int_{0}^{1 / 2} \dot{q} \dot{v} d t
\end{aligned}
$$


where we have denoted by $M$ the Lipschitz constant of $V$.

A direct evaluation of the last three integrals shows that there is a constant $\hat{C}$ such that

$$
\int_{0}^{n} L(\varphi) d t \leqslant \int_{0}^{n} L(q) d t+\hat{C}|q(n)-q(0)| .
$$

Combining this with the above estimate shows that

$$
\int_{0}^{n} L(q) d t-n c_{p} \geqslant \rho(\delta)-\hat{C}|q(n)-q(0)|,
$$

which is the desired bound.

The previous estimate has a very important consequence on the behavior of the functional $J$.

PROposition 2.7. - There exists $b \in \mathbf{R}$ such that for all $q \in$ $H_{l o c}^{1}(\mathbf{R} ; \mathbf{R}) \cap\left[u_{0}, u_{1}\right]$ and all $n \in \mathbf{N}$,

$$
\sum_{k=0}^{n-1} a_{k}(q)=\int_{0}^{n} L(q) d t-n c_{p} \geqslant b .
$$

Proof. - Notice that for all $q$ as in the statement there results $\mid q(n)-$ $q(0) \mid \leqslant \max u_{1}-\min u_{0}$. Applying Proposition 2.6 with $\delta=0$ yields

$$
\sum_{k=0}^{n-1} a_{k}(q)=\int_{0}^{n} L(q) d t-n c_{p} \geqslant-\hat{C}\left(\max u_{1}-\min u_{0}\right)=: b,
$$

which is the desired estimate.

It is convenient to retain from the preceding proposition that

$$
\liminf _{n \rightarrow+\infty} \sum_{k=-n}^{n-1} a_{k}(q) \geqslant b
$$

for all $q \in H_{l o c}^{1} \cap\left[u_{0}, u_{1}\right]$.

Remark 2.8. - We cannot hope, in general, to obtain a better estimate with $b \geqslant 0$. This is due to the fact that in the nonreversible case there 
generally exist functions $v \in H^{1}(0,1)$ such that $f(v)-c_{p}<0$. Actually, if $b$ were positive or zero, the whole problem we are dealing with could be tackled with the easier arguments from the reversible case.

We now begin the study of the behavior of the functional $J$.

LEMMA 2.9. - For all $q \in H_{l o c}^{1} \cap\left[u_{0}, u_{1}\right]$,

$$
\text { if } \limsup _{n \rightarrow+\infty} \sum_{k=-n}^{n-1} a_{k}(q)=+\infty, \quad \text { then } J(q)=+\infty,
$$

in the sense that the series $J(q)$ is well defined and diverges to $+\infty$.

Proof. - Suppose on the contrary that

$$
\liminf _{n \rightarrow+\infty} \sum_{k=-n}^{n-1} a_{k}(q)=\lambda<+\infty
$$

( $\lambda$ is finite by (11)) and choose a number $M>\lambda+1-2 b$, where $b$ is the (negative) constant defined in the previous proposition. Let $h<H$ be two positive integers such that

$$
\sum_{k=-h}^{h-1} a_{k}(q) \geqslant M \quad \text { and } \quad \sum_{k=-H}^{H-1} a_{k}(q) \leqslant \lambda+1
$$

then we have

$$
\sum_{k=-H}^{-h-1} a_{k}(q)+\sum_{k=h}^{H-1} a_{k}(q)=\sum_{k=-H}^{H-1} a_{k}(q)-\sum_{k=-h}^{h-1} a_{k}(q) \leqslant \lambda+1-M<2 b,
$$

which contradicts Proposition 2.7.

We can now prove that the functional $J$ is well defined.

Proposition 2.10. - The functional $J$ is well defined from $H_{l o c}^{1} \cap$ $\left[u_{0}, u_{1}\right]$ to $\mathbf{R} \cup\{+\infty\}$ (either the series converges or it diverges to $+\infty$ ). Moreover, if $q \in H_{l o c}^{1} \cap\left[u_{0}, u_{1}\right]$ and $J(q)<+\infty$, then $q( \pm \infty) \in \mathcal{U}$.

Proof. - We first prove the second part, namely that if $q$ does not converge to $u_{0}$ or $u_{1}$ as $t \rightarrow \pm \infty$, then $J(q)=+\infty$. We work with $t \rightarrow+\infty$. This assumption implies that there exist a number $\delta>0$ and two divergent sequences $k_{j} \in \mathbf{N}$ and $t_{j} \in\left[k_{j}, k_{j+1}\right)$ such that

$$
\left|q\left(k_{j+1}\right)-q\left(k_{j}\right)\right|=\mathrm{o}(1) \quad \text { as } j \rightarrow+\infty
$$


and

$$
\operatorname{dist}\left(q\left(t_{j}\right), \mathcal{U}\left(t_{j}\right)\right) \geqslant \delta \quad \text { for all } j \in \mathbf{N} \text {. }
$$

Relabeling, if necessary, we can assume that $\left|q\left(k_{j+1}\right)-q\left(k_{j}\right)\right|<\frac{\rho(\delta)}{2 \hat{C}}$ for all $j \in \mathbf{N}$, where $\hat{C}$ is the constant provided by Proposition 2.6. Applying this proposition in every $\left[k_{j}, k_{j+1}\right]$ we obtain

$$
\sum_{k=k_{j}}^{k_{j+1}-1} a_{k}(q) \geqslant \rho(\delta)-\hat{C}\left|q\left(k_{j+1}\right)-q\left(k_{j}\right)\right|>\frac{1}{2} \rho(\delta) .
$$

Now, recalling Proposition 2.7, we obtain, for all $n \in \mathbf{N}$,

$$
\sum_{k=-k_{n}}^{k_{n}-1} a_{k}(q) \geqslant b+\sum_{k=k_{0}}^{k_{n}-1} a_{k}(q)=b+\sum_{j=0}^{n-1} \sum_{k=k_{j}}^{k_{j+1}-1} a_{k}(q) \geqslant b+\frac{n}{2} \rho(\delta),
$$

which shows that

$$
\limsup _{n} \sum_{k=-n}^{n-1} a_{k}(q)=+\infty,
$$

and by Lemma 2.9 we conclude that $J(q)=+\infty$. This proves the second part of the Proposition.

To complete the proof it is necessary to show that $J$ is well defined (convergent or divergent to $+\infty$ ) also when $q$ tends to $u_{0}$ or $u_{1}$ as $t \rightarrow \pm \infty$. To see this, assume for definiteness that $q$ tends to $u_{0}$ and to $u_{1}$ when $t$ tends to $-\infty$ and $+\infty$ respectively (the other cases being analogous).

Working indirectly, suppose that

$$
\lambda=\liminf _{n} \sum_{k=-n}^{n-1} a_{k}(q)<\lim \sup _{n} \sum_{k=-n}^{n-1} a_{k}(q)=\Lambda
$$

and notice that we can assume $\Lambda<+\infty$, otherwise, by Lemma 2.9, $J(q)=+\infty$, and there is nothing left to prove; also, $\lambda \geqslant b$ by (11).

Let $n_{j}, m_{j}$ be two divergent sequences of positive integers such that for all $j$ there results $n_{j}+1<m_{j}<n_{j+1}-1$ and

$$
\lim _{j \rightarrow+\infty} \sum_{k=-m_{j}}^{m_{j}-1} a_{k}(q)=\lambda \quad \text { and } \quad \lim _{j \rightarrow+\infty} \sum_{k=-n_{j}}^{n_{j}-1} a_{k}(q)=\Lambda .
$$


Now as $j \rightarrow \infty$ we have

$$
\begin{aligned}
\sum_{k=-m_{j}}^{-n_{j}-1} a_{k}(q)+\sum_{k=n_{j}}^{m_{j}-1} a_{k}(q) & =\sum_{k=-m_{j}}^{m_{j}-1} a_{k}(q)-\sum_{k=-n_{j}}^{n_{j}-1} a_{k}(q) \\
& \leqslant \lambda-\Lambda+o(1)<\frac{\lambda-\Lambda}{2} .
\end{aligned}
$$

On the other hand, as $j \rightarrow \infty, q\left(-m_{j}\right)$ and $q\left(-n_{j}\right)$ tend to $u_{0}(0)$, while $q\left(m_{j}\right)$ and $q\left(n_{j}\right)$ tend to $u_{1}(0)$. Therefore, by Proposition 2.6, as $j \rightarrow \infty$ we have

$$
\begin{aligned}
& \sum_{k=-m_{j}}^{-n_{j}-1} a_{k}(q) \geqslant-\hat{C}\left|q\left(-n_{j}\right)-q\left(-m_{j}\right)\right|=\mathrm{o}(1) \\
& \quad \text { and likewise for } \sum_{k=n_{j}}^{m_{j}-1} a_{k}(q)
\end{aligned}
$$

which contradict (12). This shows that $\lambda=\Lambda$, and the proof is complete.

In the next sections we will need the following estimate of the level of functions having the same behavior at $\pm \infty$.

Proposition 2.11. - Assume $q \in H_{l o c}^{1} \cap\left[u_{0}, u_{1}\right], q \notin \mathcal{U}$, verifies $q( \pm \infty)=u_{0}$ or $q( \pm \infty)=u_{1}$. Then $J(q)>0$.

Proof. - Assume for definiteness that $q( \pm \infty)=u_{0}$. Since $q \not \equiv u_{0}$, there are $n_{0} \in \mathbf{N}$ and $\delta>0$ such that $\hat{d}\left(q_{\mid[-n, n]}, \mathcal{U}\right) \geqslant \delta$ and $\mid q(n)-$ $q(-n) \mid \leqslant \frac{\rho(\delta)}{2 \hat{C}}$ for all $n \geqslant n_{0}$. Then, by Proposition 2.6,

$$
\sum_{k=-n}^{n-1} a_{k}(q) \geqslant \rho(\delta)-\hat{C}|q(n)-q(-n)| \geqslant \frac{1}{2} \rho(\delta)
$$

for all $n \geqslant n_{0}$. This shows that $J(q)>0$.

We now establish a property that we will use in a while.

PROPOSITION 2.12. - The sublevels of $J$ in $H_{l o c}^{1} \cap\left[u_{0}, u_{1}\right]$ are bounded in $H_{l o c}^{1}$.

Proof. - Let $K \in \mathbf{R}$ and let $q \in H_{l o c}^{1} \cap\left[u_{0}, u_{1}\right]$ satisfy $J(q) \leqslant K$; clearly $\|q\|_{L^{\infty}(\mathbf{R})}$ is bounded independently of $q$. Applying Proposi- 
tion 2.7 we see that for every $n \in \mathbf{Z}$,

$$
K \geqslant J(q)=a_{n}(q)+\sum_{k<n} a_{k}(q)+\sum_{k>n} a_{k}(q) \geqslant a_{n}(q)+2 b ;
$$

therefore, recalling the definition of $a_{n}(q)$,

$$
\frac{1}{2}\|\dot{q}\|_{L^{2}(n, n+1)}^{2} \leqslant K-2 b+c_{p}+\max _{\substack{t \in[0,1] \\ x \in\left[\min u_{0}, \max u_{1}\right]}}|V(t, x)|=: \gamma .
$$

This means that $\|\dot{q}\|_{L^{2}(-n, n)}^{2} \leqslant 4 n \gamma$, which is the desired bound.

We close the section with the definition of some "cut-off" operators. To do this we first recall a "gluing lemma" from [8], to which we refer for its proof.

LEMMA 2.13. - There exist positive constants $\bar{\varepsilon}$ and $C_{1}$ such that for all $\delta \in[0, \bar{\varepsilon}]$, there exists $w \in H^{1}(0,1) \cap\left[u_{0}, u_{1}\right]$ such that

$$
\left\{\begin{array}{l}
w(0)=u_{0}(0) \\
w(1)=u_{0}(1)+\delta \\
\left|a_{0}(w)\right| \leqslant C_{1} \delta
\end{array}\right.
$$

Remark 2.14. - One can of course also show the existence of functions $w \in H^{1} \cap\left[u_{0}, u_{1}\right]$ connecting $u_{0}(0)+\delta$ to $u_{0}(1)$ or $u_{1}(0)$ to $u_{1}(1)-\delta$, or $u_{1}(0)-\delta$ to $u_{1}(1)$ with the property that $\left|a_{0}(w)\right| \leqslant C_{1} \delta$. Clearly this estimate is still true (as long as $\delta \leqslant \bar{\varepsilon}$ ) working in any $[p, p+1]$, with $p \in \mathbf{Z}$, instead of $[0,1]$, and replacing $a_{0}$ by $a_{p}$. The name " $w$ " will be reserved for this kind of functions; it is agreed that $\left|a_{p}(w)\right| \leqslant C_{1} \delta$ for all such $w$ and all $p$.

We now define (two families of) cut-off operators from $H_{l o c}^{1} \cap\left[u_{0}, u_{1}\right]$ to itself which will be used to glue a function $q$ to $u_{0}$ or $u_{1}$.

Definition 2.15. - For $i=0,1, p \in \mathbf{Z}$ and $q \in H_{l o c}^{1} \cap\left[u_{0}, u_{1}\right]$, we set

$$
\begin{aligned}
& \chi_{i}^{+}(p) q(t)= \begin{cases}q(t) & \text { if } t \leqslant p, \\
w(t) & \text { if } t \in[p, p+1], \quad \text { and } \\
u_{i}(t) & \text { if } t \geqslant p+1,\end{cases} \\
& \chi_{i}^{-}(p) q(t)= \begin{cases}u_{i}(t) & \text { if } t \leqslant p-1, \\
w(t) & \text { if } t \in[p-1, p], \\
q(t) & \text { if } t \geqslant p,\end{cases}
\end{aligned}
$$


where $w$ is, in the first case, a function that connects $q(p)$ to $u_{i}(p+1)$ in the spirit of Lemma 2.13, while $w$ connects $u_{i}(p-1)$ to $q(p)$ in the second case.

LEMMA 2.16. - Assume $q \in H_{l o c}^{1} \cap\left[u_{0}, u_{1}\right]$ is such that $J(q)$ is finite. Let $p \in \mathbf{Z}$ be a point where $\left|q(p)-u_{i}(p)\right| \leqslant \delta$, for some $i=0,1$ and some $\delta<\bar{\varepsilon}$ (this number is defined in the preceding lemma). Then

$$
\begin{aligned}
& \left|J\left(\chi_{i}^{+}(p) q\right)-\sum_{k \leqslant p-1} a_{k}(q)\right| \leqslant C_{1} \delta \quad \text { and } \\
& \left|J\left(\chi_{i}^{-}(p) q\right)-\sum_{k \geqslant p} a_{k}(q)\right| \leqslant C_{1} \delta .
\end{aligned}
$$

Proof. - It follows directly from the definition of the operators and Lemma 2.13.

\section{HETEROCLINIC SOLUTIONS}

In this section we prove the existence of the simplest type of connecting orbits, namely the heteroclinic (or one-bump) solutions between $u_{0}$ and $u_{1}$. These will be used in Section 4 to construct multibump type and chaotic trajectories.

The scheme of the proof does not differ too much from the one adopted in [16] or [8], since the technical estimates relative to the nonreversible case have been proved in Section 2.

To begin with we let

$$
\Gamma_{0}=\left\{q \in H_{l o c}^{1} \cap\left[u_{0}, u_{1}\right] / q(-\infty)=u_{0}, q(+\infty)=u_{1}\right\}
$$

be the set of functions connecting $u_{0}$ to $u_{1}$ and we denote by $\Gamma_{1}$ the analogous set of functions connecting $u_{1}$ to $u_{0}$. We also define

$$
c_{0}=\inf _{\Gamma_{0}} J \quad \text { and } \quad c_{1}=\inf _{\Gamma_{1}} J,
$$

and we notice that these numbers are both finite (see the comment following Proposition 2.7).

The next result establishes the existence of heteroclinic solutions.

THEOREM 3.1. - There exist $q_{0} \in \Gamma_{0}$ and $q_{1} \in \Gamma_{1}$ such that $J\left(q_{0}\right)=$ $c_{0}$ and $J\left(q_{1}\right)=c_{1}$. The functions $q_{0}$ and $q_{1}$ solve the equation of motion (1). 
Proof. - Assuming for a moment the existence of $q_{0}$ and $q_{1}$, we remark that the second statement of the Theorem is not trivial, since the orbits are found by constrained minimization (the constraint being $q \in\left[u_{0}, u_{1}\right]$ in the definition of the classes $\Gamma_{i}$ ). For this reason we should show that $q_{0}$ and $q_{1}$ do not touch $u_{0}$ and $u_{1}$. However this has been shown to be true in the reversible case in [8] with an argument that does not depend on reversibility and that can be repeated here without changes. We omit therefore further details on this and we concentrate on the existence of minimizing orbits.

We prove the existence of $q_{0}$. Let $q_{n} \in \Gamma_{0}$ be a minimizing sequence and let $\delta>0$ be small. Because of the boundary conditions, for every $q_{n}$ there is a unique point $t_{n}$ such that $q_{n}\left(t_{n}\right)=u_{0}\left(t_{n}\right)+\delta$ and $q_{n}(t)<$ $u_{0}(t)+\delta$ for all $t<t_{n}$. By an integer shift of the time we can achieve that $t_{n} \in[0,1)$ for every $n$; recall also that the value of $J\left(q_{n}\right)$ is not altered by integer time shifts.

The sequence $q_{n}$ is bounded in $H_{l o c}^{1}$ (Proposition 2.12) and therefore it contains a subsequence ( still denoted $q_{n}$ ) such that

$$
q_{n} \rightarrow q_{0} \quad \text { weakly in } H_{l o c}^{1} \text { and strongly in } L_{l o c}^{\infty} .
$$

We now show that $J\left(q_{0}\right)$ is convergent. To see this use Proposition 2.7 to obtain that for every $q \in H_{l o c}^{1} \cap\left[u_{0}, u_{1}\right]$ for which $J(q)$ is finite and for every $p \in \mathbf{N}$,

$$
\sum_{k=-p}^{p-1} a_{k}(q)=J(q)-\sum_{k<-p} a_{k}(q)-\sum_{k \geqslant p} a_{k}(q) \leqslant J(q)-2 b .
$$

Then by weak lower semicontinuity on bounded intervals we obtain that for every $p \in \mathbf{N}$,

$$
\sum_{k=-p}^{p-1} a_{k}\left(q_{0}\right) \leqslant \liminf _{n} \sum_{k=-p}^{p-1} a_{k}\left(q_{n}\right) \leqslant \liminf _{n}\left(J\left(q_{n}\right)-2 b\right)=c_{0}-2 b
$$

namely that $J\left(q_{0}\right)$ is convergent (apply Lemma 2.9 and Proposition 2.10). The preceding inequality also implies, via Proposition 2.10 , that the limits $q_{0}( \pm \infty)$ exist and are in $\mathcal{U}$. By construction we see that $q_{0}(-\infty)=u_{0}$, so that we must prove that $q_{0}$ has the right behavior at $+\infty$. Notice that $q_{0} \not \equiv u_{0}$ because $q_{0}\left(t^{*}\right)=u_{0}\left(t^{*}\right)+\delta$ for some $t^{*} \in[0,1]$, by our choice of $t_{n}$. 
We assume that $q_{0}(+\infty)=u_{0}$ and we show that this leads to a contradiction. Let

$$
\varepsilon=\min \left(\bar{\varepsilon}, \frac{1}{2} \frac{\rho(\delta)}{\hat{C}+C_{1}}\right),
$$

where $\hat{C}, C_{1}$ and $\bar{\varepsilon}$ are the constants provided by Proposition 2.6 and Lemma 2.13. For this $\varepsilon$ choose an integer $N \geqslant 1$ where $q_{0}(N) \leqslant u_{0}(N)+$ $\frac{\varepsilon}{2}$. By the uniform convergence of $q_{n}$, for all $n$ large enough we have $q_{n}(N) \leqslant u_{0}(N)+\varepsilon$.

Now, since $q_{n}(-\infty)=u_{0}$ for all $n$, we see that for all integers $p$ less than a convenient $p_{n}$, there results

$$
\left|q_{n}(p)-q_{n}(N)\right| \leqslant \varepsilon .
$$

Moreover, since $q_{n}\left(t_{n}\right)=u_{0}\left(t_{n}\right)+\delta$ for some $t_{n} \in[0,1)$ and all $n$, we also have that

$$
\hat{d}\left(q_{n \mid[p, N]}, \mathcal{U}\right) \geqslant \delta
$$

for all $p \leqslant p_{n}$ and all large $n$. This means, by Proposition 2.6, that

$$
\sum_{k=p}^{N-1} a_{k}\left(q_{n}\right) \geqslant \rho(\delta)-\hat{C} \varepsilon,
$$

and, this being true for all $p \leqslant p_{n}$, we finally obtain

$$
\sum_{k<N} a_{k}\left(q_{n}\right) \geqslant \rho(\delta)-\hat{C} \varepsilon
$$

for every large $n \in \mathbf{N}$. Now, recalling Lemma 2.16, we evaluate

$$
\begin{aligned}
J\left(\chi_{0}^{-}(N) q_{n}\right) & \leqslant \sum_{k \geqslant N} a_{k}\left(q_{n}\right)+C_{1} \varepsilon=J\left(q_{n}\right)-\sum_{k<N} a_{k}\left(q_{n}\right)+C_{1} \varepsilon \\
& \leqslant J\left(q_{n}\right)-\rho(\delta)+\hat{C} \varepsilon+C_{1} \varepsilon=J\left(q_{n}\right)-\frac{1}{2} \rho(\delta)<c_{0}
\end{aligned}
$$

for $n$ large. Since $\chi_{0}^{-}(N) q_{n} \in \Gamma_{0}$, this is a contradiction, and our claim is proved.

Finally we prove that $J\left(q_{0}\right)=c_{0}$. Suppose this is false; then $J\left(q_{0}\right)=$ $c_{0}+\sigma$ for some $\sigma>0$. Let $\varepsilon<\frac{\sigma}{16 \hat{C}}$ and take $p_{\varepsilon} \in \mathbf{N}$ such that

$$
q_{0}(-p) \leqslant u_{0}(-p)+\varepsilon \quad \text { and } q_{0}(p) \geqslant u_{1}(p)-\varepsilon
$$

for all integers $p \geqslant p_{\varepsilon}$. 
It is not restrictive to assume that also

$$
\sum_{k=-p}^{p-1} a_{k}\left(q_{0}\right) \geqslant c_{0}+\frac{3}{4} \sigma \quad \text { for all } p \geqslant p_{\varepsilon} .
$$

We now choose a $p \geqslant p_{\varepsilon}$ and we notice that since

$$
\sum_{k=-p}^{p-1} a_{k}\left(q_{0}\right) \leqslant \liminf _{n} \sum_{k=-p}^{p-1} a_{k}\left(q_{n}\right),
$$

we can fix $n_{0}$ such that

$$
\sum_{k=-p}^{p-1} a_{k}\left(q_{n}\right) \geqslant \sum_{k=-p}^{p-1} a_{k}\left(q_{0}\right)-\frac{\sigma}{4} \text { for all } n \geqslant n_{0} .
$$

At the same time we can also think that $n_{0}$ is so large that

$$
\begin{aligned}
& q_{n}(-p) \leqslant u_{0}(-p)+2 \varepsilon \text { and } q_{n}(p) \geqslant u_{1}(p)-2 \varepsilon \\
& \quad \text { for all } n \geqslant n_{0} .
\end{aligned}
$$

Now, by Proposition 2.6,

$$
\begin{aligned}
& \sum_{k<-p} a_{k}\left(q_{n}\right) \geqslant-2 \hat{C} \varepsilon \text { and } \sum_{k \geqslant p} a_{k}\left(q_{n}\right) \geqslant-2 \hat{C} \varepsilon \\
& \quad \text { for all } n \geqslant n_{0} .
\end{aligned}
$$

Combining this and the above estimate we see that for all $n \geqslant n_{0}$

$$
\begin{aligned}
J\left(q_{n}\right) & \geqslant-2 \hat{C} \varepsilon+\sum_{k=-p}^{p-1} a_{k}\left(q_{n}\right)-2 \hat{C} \varepsilon \geqslant \sum_{k=-p}^{p-1} a_{k}\left(q_{0}\right)-\frac{\sigma}{4}-4 \hat{C} \varepsilon \\
& \geqslant c_{0}+\frac{\sigma}{4}
\end{aligned}
$$

by (13) and our choice of $\varepsilon$. This contradicts the fact that $J\left(q_{n}\right)$ tends to $c_{0}$, and the proof is complete.

The orbits $q_{0}$ and $q_{1}$ satisfy the following monotonicity property.

COROLlary 3.2. - Let $q_{0} \in \Gamma_{0}$ and $q_{1} \in \Gamma_{1}$ be the solutions found in Theorem 3.1. Then

$$
q_{0}(t)<q_{0}(t+1) \quad \text { and } \quad q_{1}(t)>q_{1}(t+1) \quad \text { for all } t \in \mathbf{R} \text {. }
$$


Proof. - We prove the inequality concerning $q_{0}$. To this aim, assume that at some $t_{0} \in \mathbf{R}$ there results $q_{0}\left(t_{0}\right) \geqslant q_{0}\left(t_{0}+1\right)$. Then pick $t_{1}>t_{0}$ where $q_{0}\left(t_{1}\right)<q_{0}\left(t_{1}+1\right)$, which exists since $q_{0}(+\infty)=u_{1}$. Defining $g(t)=q_{0}(t+1)-q_{0}(t)$ on $\left[t_{0}, t_{1}\right]$, we see that $g$ must vanish at some point $t^{*} \in\left[t_{0}, t_{1}\right]$. Setting

$$
\bar{q}(t)= \begin{cases}q_{0}(t) & \text { if } t \leqslant t^{*}, \\ q_{0}(t+1) & \text { if } t \geqslant t^{*},\end{cases}
$$

it is easy to check that $J(\bar{q})<J\left(q_{0}\right)$, since we have taken out of $q_{0}$ a 1 -periodic function away from $\mathcal{U}$. This is impossible, and the inequality is proved.

Remark 3.3. - We observe that the solutions found by means of the preceding Theorem also verify natural boundary conditions on the derivatives. Stating it only for $q_{0}$, we have

$$
\lim _{t \rightarrow-\infty}\left(\dot{q}_{0}(t)-\dot{u}_{0}(t)\right)=\lim _{t \rightarrow+\infty}\left(\dot{q}_{0}(t)-\dot{u}_{1}(t)\right)=0 .
$$

This can be easily proved exactly like in [8], Corollary 3.10.

Remark 3.4. - The sign of $c_{0}$ and $c_{1}$ is unknown; however we can say that $c_{0}+c_{1}>0$. To see this let $t^{*}$ be a point where $q_{0}\left(t^{*}\right)=q_{1}\left(t^{*}\right)$ (which exists because of the boundary conditions) and let

$$
q^{-}(t)=\left\{\begin{array}{ll}
q_{0}(t) & \text { if } t \leqslant t^{*}, \\
q_{1}(t) & \text { if } t \geqslant t^{*},
\end{array} \quad \text { and } \quad q^{+}(t)= \begin{cases}q_{1}(t) & \text { if } t \leqslant t^{*} \\
q_{0}(t) & \text { if } t \geqslant t^{*}\end{cases}\right.
$$

Then, as it is readily seen, $J\left(q_{0}\right)+J\left(q_{1}\right)=J\left(q^{-}\right)+J\left(q^{+}\right)$, so that $c_{0}+c_{1}=J\left(q^{-}\right)+J\left(q^{+}\right)>0$ because of Proposition 2.11.

Remark 3.5. - The fact that one between $c_{0}$ and $c_{1}$ may be negative affects the semicontinuity properties of $J$. Indeed, assume for instance that $c_{1}<0$, let $t_{n}$ be the largest time where $q_{0}\left(t_{n}\right)=q_{1}\left(t_{n}-n\right)$ and set

$$
\bar{q}_{n}(t)= \begin{cases}q_{0}(t) & \text { if } t \leqslant t_{n} \\ q_{1}(t-n) & \text { if } t \geqslant t_{n}\end{cases}
$$

Then it is easy to see that $J\left(\bar{q}_{n}\right)=c_{0}+c_{1}+o(1)$ as $n \rightarrow+\infty$ and that $\bar{q}_{n}$ tends weakly to $q_{0}$ in $H_{l o c}^{1}$. Therefore

$$
J\left(q_{0}\right)=c_{0}>c_{0}+c_{1}=\liminf _{n} J\left(\bar{q}_{n}\right),
$$

and $J$ is not (sequentially) weakly lower semicontinuous. 


\section{MULTIBUMP-TYPE AND CHAOTIC ORBITS}

The construction of multibump and chaotic solutions for Eq. (1) is based on a shadowing-like argument in the spirit of [18,8] and [1], where it was carried out in the reversible case. It requires a suitable adaptation of a nondegeneracy condition which has been introduced in [8] and that we are now going to state. First of all we describe roughly what we mean by multibump solutions (a precise definition can be found in the statement of the theorems). We use this term to indicate solutions that, given a number $\delta>0$, are alternately "closer than $\delta$ " to the periodic states $u_{0}$ and $u_{1}$ on prescribed time intervals, and are asymptotic to $u_{0}$ or $u_{1}$ as $t \rightarrow \pm \infty$. We consider as a "bump" here the change of state of an orbit (from close to $u_{0}$ to close to $u_{1}$, for example). The asymptotic behavior of a multibump solution $q$ is obtained by imposing the desired values of $q( \pm \infty)$ in $\mathcal{U}$.

We now turn to the description of the nondegeneracy condition. That such a condition is necessary to obtain a rich structure of solutions is evident from the fact that no multibump solution is present in autonomous scalar problems. This is of course false for autonomous systems; see, e.g., [6] for such a case. This condition is therefore strictly related to the explicit time dependence in the equation and replaces the classical assumptions on the intersection of the stable and unstable manifolds of $u_{0}$ and $u_{1}$ that are used in the perturbative approach (see for example [23, $3]$ ). Roughly it says that there must not be too many heteroclinics between $u_{0}$ and $u_{1}$. To state it precisely, let

$$
\begin{aligned}
& \mathcal{S}_{0}=\left\{q(0) / q \in \Gamma_{0}, J(q)=c_{0}\right\} \quad \text { and } \\
& \mathcal{S}_{1}=\left\{q(0) / q \in \Gamma_{1}, J(q)=c_{1}\right\} .
\end{aligned}
$$

These are infinite subsets of the interval $\left(u_{0}(0), u_{1}(0)\right)$ which accumulate at its boundary points (to see this examine the sequences $q_{0}(k)$ and $q_{1}(k)$ with $k \in \mathbf{Z}$ ); if the problem is autonomous, clearly $\mathcal{S}_{0}=\mathcal{S}_{1}=$ $\left(u_{0}(0), u_{1}(0)\right)$.

The condition we require from now on is the following.

$$
\mathcal{S}_{0} \neq\left(u_{0}(0), u_{1}(0)\right) \quad \text { and } \quad \mathcal{S}_{1} \neq\left(u_{0}(0), u_{1}(0)\right) \text {. }
$$

In Section 5 we analyze the relationship between $(*)$ and the classical conditions; for our present purposes we only make use of the following important consequence of $(*)$.

Proposition 4.1. - If $(*)$ holds, then for every $\hat{\delta}>0$ there exist $\delta_{i} \in(0, \hat{\delta}), i=0, \ldots, 3$ and two positive numbers $\Lambda_{0}, \Lambda_{1}$ such that 


$$
\begin{aligned}
& \inf \left\{J(q) / q \in \Gamma_{0}, q(0)=u_{0}(0)+\delta_{0}, \text { or } q(0)=u_{1}(0)-\delta_{2}\right\} \\
& \quad \geqslant c_{0}+\Lambda_{0}
\end{aligned}
$$

and

$$
\begin{aligned}
& \inf \left\{J(q) / q \in \Gamma_{1}, q(0)=u_{1}(0)-\delta_{1}, \text { or } q(0)=u_{0}(0)+\delta_{3}\right\} \\
& \quad \geqslant c_{1}+\Lambda_{1} .
\end{aligned}
$$

We will use condition $(*)$ in the sense given by the previous proposition, namely, there are points as close as we wish to $u_{0}(0)$ and to $u_{1}(0)$ through which no minimizing heteroclinic can pass. The proof of Proposition 4.1 can be found in [8] or [18] in the reversible case. Since the argument used in those papers does not depend on reversibility it can be repeated here without changes, and therefore we omit it.

We now proceed to the first step in the construction of multibump solutions. To this end, we begin by fixing some constants.

DEFINITION 4.2. - We set

$$
\bar{d}=\frac{1}{2} \min _{t}\left(u_{1}(t)-u_{0}(t)\right)
$$

and we fix a positive number $\hat{\delta}$ such that

$$
\hat{\delta}<\min \left(\bar{\varepsilon}, \bar{d}, \frac{\rho(\bar{d})}{\hat{C}+2 C_{1}}\right)
$$

where $\rho, \hat{C}, C_{1}$ and $\bar{\varepsilon}$ are defined in Propositions 2.5, 2.6 and Lemma 2.13.

Let now $\hat{\delta}$ be as in the previous definition, take $\delta_{0}, \ldots, \delta_{3}$ as in Proposition 4.1, and let $m \in \mathbf{N}$. We define the sets

$$
\begin{gathered}
X_{0}=\left\{q \in H_{l o c}^{1} \cap\left[u_{0}, u_{1}\right] / q(-m) \leqslant u_{0}(-m)+\delta_{0}\right. \\
\text { and } \left.q(m) \geqslant u_{1}(m)-\delta_{2}\right\}
\end{gathered}
$$

and

$$
\begin{gathered}
X_{1}=\left\{q \in H_{l o c}^{1} \cap\left[u_{0}, u_{1}\right] / q(-m) \geqslant u_{1}(-m)-\delta_{1}\right. \\
\text { and } \left.q(m) \leqslant u_{0}(m)+\delta_{3}\right\} .
\end{gathered}
$$

These sets depend on $m$, a constant which will be appropriately fixed later. For the moment we will agree that $m$ is large enough so that $X_{0}$ and $X_{1}$ contain two heteroclinic solutions $q_{0} \in \Gamma_{0}$ and $q_{1} \in \Gamma_{1}$ respectively. 
The multibump solutions we are interested in will be found among orbits which "look like" gluing of translates of functions in $X_{0}$ and $X_{1}$. The argument will once again be minimization of $J$ over suitable sets of functions. The main problem is to show that the minimizers are free from the constraint imposed as in $X_{0}$ and $X_{1}$.

Remark 4.3. - Some notation conventions are in order. From now on we will agree that when we label $c, \Lambda, u, q, \Gamma, X$ and $\chi^{ \pm}$with an index, that index is considered mod 2, so that for example $q_{i}$ is $q_{0}$ if $i$ is even, and $q_{1}$ otherwise. This will simplify some formulas.

Keeping this in mind we now define some subsets of $H_{l o c}^{1} \cap\left[u_{0}, u_{1}\right]$ which we will need to construct multibump solutions.

Definition 4.4. - Let $P=\left(p_{i}\right)_{i \in \mathbf{Z}} \subset \mathbf{Z}$ be a bi-infinite set of integers such that $p_{i+1}-p_{i} \geqslant 4 m$ for all $i$. We set, for every pair of integers $j \leqslant k$,

$$
\begin{aligned}
& \Gamma(j, k)=\left\{q \in H_{l o c}^{1} \cap\left[u_{0}, u_{1}\right] / q(-\infty)=u_{j}, q(+\infty)=u_{k+1},\right. \\
&\left.q\left(\cdot+p_{i}\right) \in X_{i} \forall i=j, \ldots, k\right\}
\end{aligned}
$$

and

$$
c(j, k)=\inf _{\Gamma(j, k)} J .
$$

Remark 4.5. - From now on we consider the set $P$ as fixed. Notice that functions in $\Gamma(j, k)$ satisfy "natural" boundary conditions with respect to the constraints. For instance, if $j$ is even, then any $q \in \Gamma(j, k)$ is close to $u_{0}$ at $t=p_{j}-m$ and it is required to be asymptotic to $u_{0}$ at $-\infty$. Notice also the particular case $c(j, j)=c_{j}$ (recall Remark 4.3).

The first step in the construction of multibump solution is to show that the levels $c(j, k)$ are attained.

Proposition 4.6. - Let $P \subset \mathbf{Z}$ be as above and let $j \leqslant k$ be integers. Then there exists $q_{j k} \in \Gamma(j, k)$ such that $J\left(q_{j k}\right)=c(j, k)$.

Proof. - It is a variant of the proof of Theorem 3.1. If $j=k$, the statement follows trivially from the fact that in this case the minimizers are $q_{0}$ and $q_{1}$. Let therefore $j<k$ and consider a minimizing sequence $q_{n} \in \Gamma(j, k)$ for $J$. Then, by Proposition 2.12, $q_{n}$ is bounded in $H_{l o c}^{1}$, and therefore it possesses a subsequence, still denoted $q_{n}$ such that

$$
q_{n} \rightarrow q_{j k} \quad \text { weakly in } H_{l o c}^{1} \text { and strongly in } L_{l o c}^{\infty} .
$$


Clearly $q_{j k}$ verifies the constraints at $p_{i} \pm m$ for all $i=j, \ldots, k$. To prove that $q_{j k} \in \Gamma(j, k)$ we must show that $q_{j k}$ satisfies the appropriate conditions at $\pm \infty$. Before doing this we notice that as in the proof of Theorem 3.1, $J(q)$ is finite, so that by Proposition 2.10, the limits $q( \pm \infty)$ exist and are in $\mathcal{U}$. We carry out the details only at $+\infty$, and assuming that $k$ is odd (the other cases being similar). If $k$ is odd, then $q_{j k}\left(p_{k}+m\right) \leqslant u_{0}\left(p_{k}+m\right)+\delta_{3}$ and we must check that $q_{j k}(+\infty)=u_{0}$. Arguing indirectly, we assume that $q_{j k}(+\infty)=u_{1}$ and we show that this leads to a contradiction.

Choose an integer $N>p_{k}+m$ so that

$$
\hat{d}\left(q_{j k \mid\left[p_{k}+m, N\right]}, \mathcal{U}\right)>\bar{d},
$$

which is possible since $q_{j k}(+\infty)=u_{1}$. This inequality also holds for $q_{n}$, as soon as $n$ is large enough, because of $L_{\text {loc }}^{\infty}$ convergence. Moreover, since $q_{n} \in \Gamma(j, k)$, there results $q_{n}(p) \leqslant u_{0}(p)+\delta_{3}$ for all integers $p$ larger than some $p_{n}$. This means that $\left|q_{n}(p)-q_{n}\left(p_{k}+m\right)\right| \leqslant \delta_{3}$ for all large $p$, so that the application of Proposition 2.6 to $q_{n}$ on all intervals $\left[p_{k}+m, p\right]$ yields

$$
\sum_{i \geqslant p_{k}+m} a_{i}\left(q_{n}\right) \geqslant \rho(\bar{d})-\hat{C} \delta_{3} .
$$

Recalling Lemma 2.16 we evaluate, as $n \rightarrow+\infty$,

$$
\begin{aligned}
J\left(\chi_{0}^{+}\left(p_{k}+m\right) q_{n}\right) & \leqslant \sum_{i<p_{k}+m} a_{i}\left(q_{n}\right)+C_{1} \delta_{3} \\
& =J\left(q_{n}\right)-\sum_{i \geqslant p_{k}+m} a_{i}\left(q_{n}\right)+C_{1} \delta_{3} \\
& \leqslant J\left(q_{n}\right)-\rho(\bar{d})+\left(\hat{C}+C_{1}\right) \delta_{3} \\
& =c(j, k)-\rho(\bar{d})+\left(\hat{C}+C_{1}\right) \delta_{3}+\mathrm{o}(1) .
\end{aligned}
$$

Since $\chi_{0}^{+}\left(p_{k}+m\right) q_{n} \in \Gamma(j, k)$, this contradicts the definition of $c(j, k)$ (recall Definition 4.2); therefore $q_{j k}(+\infty)=u_{0}$.

To complete the proof we should also check that $J\left(q_{j k}\right)=c(j, k)$; this can be done exactly as in the proof of Theorem 3.1, and we do not repeat the details.

The functions $q_{j k}$ found in the previous proposition do not, in general, solve Eq. (1). This is due to the constrains imposed at the points $p_{i} \pm m$. In the remaining part of this section we prove that by appropriately choosing 
$m$, the minimizers $q_{j k}$ are free from constraints, so that they solve the problem in a classical sense. In order to do this, and also in order to obtain estimates independent of the number of points $p_{i}$, some care must be taken.

Definition 4.7. - We choose a positive $\varepsilon<\bar{\varepsilon}(\bar{\varepsilon}$ is defined in Lemma 2.13) such that

$$
\varepsilon<\frac{\min \left(\Lambda_{0}, \Lambda_{1}\right)}{4 C_{1}}
$$

and we require from now on that the number $m$ used to define $P$ is large enough so that the following inequalities hold:

$$
\begin{aligned}
& q_{0}(2 m)>u_{1}(2 m)-\delta_{1} \quad \text { and } \quad q_{0}(-2 m)<u_{0}(-2 m)+\delta_{3}, \\
& q_{1}(2 m)<u_{0}(2 m)+\delta_{0} \quad \text { and } \quad q_{1}(-2 m)>u_{1}(-2 m)-\delta_{2}
\end{aligned}
$$

and

$$
m \geqslant \frac{2 C_{1} \hat{\delta}}{\rho(\varepsilon)} .
$$

The following proposition establishes a key inequality for the rest of the argument. Here we only need that $m$ is chosen so that (19) and (20) hold.

Proposition 4.8. - Let $j \leqslant l<k$ be integers. Then

$$
c(j, k)<c(j, l)+c(l+1, k) .
$$

Proof. - We only prove the proposition for $l$ even, the other case being analogous. Let $q_{j l}$ and $q_{l+1, k}$ be minimizers of $J$ over $\Gamma(j, l)$ and $\Gamma(l+1, k)$ respectively. We claim that it is not restrictive to assume that

$$
\begin{gathered}
q_{j l}\left(p_{l+1}-m\right)>u_{1}\left(p_{l+1}-m\right)-\delta_{1}, \\
q_{l+1, k}\left(p_{l}+m\right)>u_{1}\left(p_{l}+m\right)-\delta_{2},
\end{gathered}
$$

in the sense that we can choose minimizers $q_{j l}$ and $q_{l+1, k}$ satisfying these requirements. Indeed, if $l=j$, this is certainly true by taking as $q_{j l}$ the function $q_{0}\left(\cdot-p_{l}\right)$ : recalling the monotonicity property of Corollary 3.2, we obtain 


$$
\begin{aligned}
q_{j l}\left(p_{l+1}-m\right) & =q_{0}\left(p_{l+1}-m-p_{l}\right)>q_{0}(2 m)>u_{1}(2 m)-\delta_{1} \\
& =u_{1}\left(p_{l+1}-m\right)-\delta_{1}
\end{aligned}
$$

by our choice of the points in $P$ and (19). In the same way one can check (23) when $l=k-1$. Proving (22) when $l>j$ is more delicate.

Let $\bar{q}=q_{0}\left(\cdot-p_{l}\right)$. Without being too precise, we note that $\bar{q}$ is close to $u_{0}$ at $p_{l-1}-m$ and verifies $\bar{q}\left(p_{l+1}-m\right)>u_{1}\left(p_{l+1}-m\right)-\delta_{1}$. On the other hand, $q_{j l}$ is close to $u_{1}$ at $p_{l-1}-m$; if (22) is violated, then we see that $\bar{q}$ and $q_{j l}$ must cross at some $t^{*} \in\left[p_{l-1}-m, p_{l+1}-m\right]$. Set

$$
q^{+}(t)=\left\{\begin{array}{ll}
q_{j l}(t) & \text { if } t \leqslant t^{*}, \\
\bar{q}(t) & \text { if } t \geqslant t^{*},
\end{array} \quad \text { and } \quad q^{-}(t)= \begin{cases}\bar{q}(t) & \text { if } t \leqslant t^{*} \\
q_{j l}(t) & \text { if } t \geqslant t^{*}\end{cases}\right.
$$

and observe that $q^{+} \in \Gamma(j, l)$ and satisfies (22), and $q^{-} \in \Gamma_{0}$. Now, as it is easily seen,

$$
J\left(q^{+}\right) \leqslant J\left(q^{+}\right)+J\left(q^{-}\right)-c_{0}=J\left(q_{j l}\right)+J(\bar{q})-c_{0}=c(j, l),
$$

so that we can replace $q_{j l}$ by $q^{+}$and get (22). In a similar way one can prove (23) when $l<k-1$.

To complete the proof we use $q_{j l}$ and $q_{l+1, k}$ to estimate $c(j, k)$. Using arguments similar to those in the first part of the proof, it is not difficult to show that $q_{j l}$ and $q_{l+1, k}$ must cross at some $t^{*} \in\left[p_{l}-m, p_{l+1}+m\right]$. Setting this time

$$
q^{-}(t)=\left\{\begin{array}{ll}
q_{j l}(t) & \text { if } t \leqslant t^{*}, \\
q_{l+1, k}(t) & \text { if } t \geqslant t^{*},
\end{array} \quad \text { and } \quad q^{+}(t)= \begin{cases}q_{l+1, k}(t) & \text { if } t \leqslant t^{*} \\
q_{j l}(t) & \text { if } t \geqslant t^{*}\end{cases}\right.
$$

we see that $q^{-} \in \Gamma(j, k)$ (because it satisfies the right constraints at $p_{l}+m$ and $p_{l+1}-m$ by (22), (23)) and that $q^{+}( \pm \infty)=u_{1}$, so that $J\left(q^{+}\right)>0$ by Proposition 2.11. From this we obtain

$$
J\left(q^{-}\right)<J\left(q^{-}\right)+J\left(q^{+}\right)=J\left(q_{j l}\right)+J\left(q_{l+1, k}\right)=c(j, l)+c(l+1, k),
$$

from which the desired inequality follows at once.

We finally show that the minimizers $q_{j k}$ found by Proposition 4.6 provide the solutions we seek. The last step consists in showing that the functions $q_{j k}$ are "free from constraints".

The next proposition completes therefore the proof of the existence of multibump solutions. 
Proposition 4.9. - Assume (*) holds and let $m$ be fixed as in Definition 4.7. For all integers $j<k$, let $q_{j k} \in \Gamma(j, k)$ be the minimizer found in Proposition 4.6. Then $q_{j k}$ is free from constraints, in the sense that

$$
\begin{gathered}
u_{0}(t)<q_{j k}(t)<u_{1}(t) \quad \text { for all } t \in \mathbf{R}, \\
q_{j k}\left(p_{i}-m\right)<u_{0}\left(p_{i}-m\right)+\delta_{0} \quad \text { and } \\
q_{j k}\left(p_{i}+m\right)>u_{1}\left(p_{i}+m\right)-\delta_{2}, \quad i \text { even, } \\
q_{j k}\left(p_{i}-m\right)>u_{1}\left(p_{i}-m\right)-\delta_{1} \quad \text { and } \\
q_{j k}\left(p_{i}+m\right)<u_{0}\left(p_{i}+m\right)+\delta_{3}, \quad i \text { odd }
\end{gathered}
$$

for all $j \leqslant i \leqslant k$. In particular, therefore, $q_{j k}$ solves $E q$. (1).

Proof. - The strict inequality (24) is a consequence of the minimizing properties of $u_{0}, u_{1}$ and $q_{j k}$; we omit its proof, as we did in Theorem 3.1, since the details can be found in [8]. To prove the other inequalities we set $q=q_{j k}$ for notational convenience. We first show that

$$
\sum_{h=p_{i}+m}^{p_{i+1}-m-1} a_{h}(q) \leqslant 2 C_{1} \hat{\delta} \quad \text { for } i=j, \ldots, k-1 .
$$

To see this, let $w_{1}$ be a function that connects $q\left(p_{i}+m\right)$ to $u_{i+1}\left(p_{i}+\right.$ $m+1)$ in the spirit of Lemma 2.13, and likewise, let $w_{2}$ be a function that connects $u_{i+1}\left(p_{i+1}-m-1\right)$ to $q\left(p_{i+1}-m\right)$. Setting

$$
\bar{u}= \begin{cases}w_{1} & \text { in }\left[p_{i}+m, p_{i}+m+1\right], \\ u_{i+1} & \text { in }\left[p_{i}+m+1, p_{i+1}-m-1\right], \\ w_{2} & \text { in }\left[p_{i+1}-m-1, p_{i+1}-m\right],\end{cases}
$$

we see that

$$
\sum_{h=p_{i}+m}^{p_{i+1}-m-1} a_{h}(q) \leqslant \sum_{h=p_{i}+m}^{p_{i+1}-m-1} a_{h}(\bar{u}) \leqslant 2 C_{1} \hat{\delta},
$$

because $q$ is a minimizer; this proves (25).

Let now $I_{i}=\left[p_{i}+m, p_{i+1}-m\right]$. We prove that there exists $n_{i} \in I_{i} \cap \mathbf{Z}$ such that

$$
\left|q\left(n_{i}\right)-u_{i+1}\left(n_{i}\right)\right|<\varepsilon \quad \text { for all } i=j, \ldots, k-1,
$$


where $\varepsilon$ is the constant introduced in Definition 4.7. First of all, we observe that $q(t) \neq \frac{1}{2}\left(u_{1}(t)+u_{0}(t)\right)$ for all $t \in I_{i}$. Indeed, if this is not the case, then $\hat{d}\left(q_{\mid I_{i}}, \mathcal{U}\right) \geqslant \bar{d}$, and, by Proposition 2.6,

$$
\sum_{h=p_{i}+m}^{p_{i+1}-m-1} a_{h}(q) \geqslant \rho(\bar{d})-\hat{C}\left|q\left(p_{i+1}-m\right)-q\left(p_{i}+m\right)\right| \geqslant \rho(\bar{d})-\hat{C} \hat{\delta}
$$

contradicting, via (25), the choice of $\hat{\delta}$ made in (16).

Keeping in mind the inequalities that $q$ satisfies at the endpoints of $I_{i}$, we therefore see that

$$
\left|q(t)-u_{i}(t)\right|>\frac{1}{2}\left(u_{1}(t)-u_{0}(t)\right) \quad \text { for all } t \in I_{i}
$$

so that $\hat{d}\left(q_{\mid I_{i}}, \mathcal{U}\right)=\left\|q-u_{i+1}\right\|_{L^{\infty}\left(I_{i}\right)}$ and

$$
\operatorname{dist}(q(n), \mathcal{U}(n))=\left|q(n)-u_{i+1}(n)\right| \quad \text { for all } n \in I_{i} \cap \mathbf{Z} \text {. }
$$

Assume now that (26) is violated, so that for some $j \leqslant i \leqslant k-1$ there results

$$
\operatorname{dist}(q(n), \mathcal{U}(n))=\left|q(n)-u_{i+1}(n)\right| \geqslant \varepsilon \quad \text { for all } n \in I_{i} \cap \mathbf{Z} \text {. }
$$

Let $\bar{q}=\chi_{i+1}^{-}\left(p_{i}+m\right) \circ \chi_{i+1}^{+}\left(p_{i+1}-m\right) q$ restricted to the interval $\left[p_{i}+\right.$ $\left.m-1, p_{i+1}-m+1\right]$; then $\bar{q}\left(p_{i}+m-1\right)=\bar{q}\left(p_{i+1}-m+1\right)$, and, by (27),

$$
\operatorname{dist}(\bar{q}(n), \mathcal{U}(n)) \geqslant \varepsilon \quad \text { for all } n \in I_{i} \cap \mathbf{Z} .
$$

Applying Proposition 2.5 to $\bar{q}$ yields

$$
\begin{aligned}
\sum_{h=p_{i}+m}^{p_{i+1}-m-1} a_{h}(q) & \geqslant \sum_{h=p_{i}+m-1}^{p_{i+1}-m} a_{h}(\bar{q})-2 C_{1} \hat{\delta} \\
& \geqslant\left(p_{i+1}-p_{i}-2 m+2\right) \rho(\varepsilon)-2 C_{1} \hat{\delta},
\end{aligned}
$$

which, taken (25) into account, violates the choice of $m$ made in (21) (recall also that $p_{i+1}-p_{i} \geqslant 4 m$ ). We have thus proved the existence of the points $n_{i}$ satisfying (26).

To complete the proof we first argue in the case $i \neq j, k$. We let $q_{l}=\chi_{i}^{+}\left(n_{i-1}\right) q, q_{r}=\chi_{i+1}^{-}\left(n_{i}\right) q$ and $q_{c}=\chi_{i}^{-}\left(n_{i-1}\right) \circ \chi_{i+1}^{+}\left(n_{i}\right) q$. Notice that

$$
q_{l} \in \Gamma(j, i-1), \quad q_{r} \in \Gamma(i+1, k) \quad \text { and } \quad q_{c} \in \Gamma_{i} .
$$


Assume now that $q$, and therefore $q_{c}$ violates one of the inequalities in the statement of the proposition. Then, by Proposition 4.1,J $\left(q_{c}\right) \geqslant c_{i}+\Lambda_{i}$. Hence, by Proposition 4.8,

$$
\begin{aligned}
c_{i}+\Lambda_{i} & \leqslant J\left(q_{c}\right) \leqslant J(q)-J\left(q_{l}\right)-J\left(q_{r}\right)+4 C_{1} \varepsilon \\
& \leqslant c(j, k)-c(j, i-1)-c(i+1, k)+4 C_{1} \varepsilon \\
& <c(i, i)+4 C_{1} \varepsilon=c_{i}+4 C_{1} \varepsilon,
\end{aligned}
$$

which contradicts the choice of $\varepsilon$ in (18).

Finally, if $i=j$, one simply chooses a point $n_{j-1}$ so close to $-\infty$ that $\left|q\left(n_{j-1}\right)-u_{j}\left(n_{j-1}\right)\right|<\varepsilon$ (this point exists because $q(-\infty)=u_{j}$ ) and repeats the above argument with the only change that now $J\left(q_{l}\right)>0$ because $q_{l}( \pm \infty)=u_{j}$. Obvious modifications when $i=k$. The proof is complete.

We summarize the results obtained so far in a single theorem. In its statement recall the "mod 2" convention (Remark 4.3).

THEOREM 4.10. - Assume $u_{0}$ and $u_{1}$ are consecutive minimizers and let $(*)$ hold. Then for every $\delta>0$ small enough there exists $m=m(\delta) \in \mathbf{N}$ such that for every sequence $\left(p_{i}\right)_{i \in \mathbf{Z}} \subset \mathbf{Z}$ such that $p_{i+1}-p_{i} \geqslant 4 m$ and for every $j, k \in \mathbf{Z}$ with $j<k$, there exists a classical solution $q$ of (1) satisfying

$$
\begin{gathered}
u_{0}(t)<q(t)<u_{1}(t) \quad \text { for all } t \in \mathbf{R}, \\
q(-\infty)=u_{j}, \quad q(+\infty)=u_{k+1}
\end{gathered}
$$

and, for all $i=j, \ldots, k$,

$$
\begin{aligned}
& \left|q\left(p_{i}-m\right)-u_{i}\left(p_{i}-m\right)\right| \leqslant \delta \quad \text { and } \\
& \left|q\left(p_{i}+m\right)-u_{i+1}\left(p_{i}+m\right)\right| \leqslant \delta .
\end{aligned}
$$

Remark 4.11. - The theorem establishes the existence of multibump solutions. Its statement could be made more precise, in the spirit of the shadowing lemma, in the sense that solutions $q$ could be found satisfying the inequality $\left|q(t)-u_{i+1}(t)\right| \leqslant \delta$ in the whole intervals $\left[p_{i}+m, p_{i+1}-m\right]$; to achieve this one must only choose a bit more carefully the various constants used in the proof. We won't detail this point.

A great deal of technicalities in the proof of Theorem 4.10 have been introduced in order to obtain estimates independent of the number of bumps. This allows us, with a classical argument, to establish the 
existence of solutions having infinitely many bumps, which give further evidence of the chaotic nature of the dynamics associated to Eq. (1).

THEOREM 4.12. - Assume $u_{0}$ and $u_{1}$ are consecutive minimizers and let $(*)$ hold. Then for every $\delta>0$ small enough there exists $m=m(\delta) \in \mathbf{N}$ such that for every sequence $\left(p_{i}\right)_{i \in \mathbf{Z}} \subset \mathbf{Z}$ such that $p_{i+1}-p_{i} \geqslant 4 m$ there exists a classical solution $q$ of (1) satisfying

$$
u_{0}(t)<q(t)<u_{1}(t) \quad \text { for all } t \in \mathbf{R},
$$

and

$$
\begin{aligned}
& \left|q\left(p_{i}-m\right)-u_{i}\left(p_{i}-m\right)\right| \leqslant \delta \quad \text { and } \\
& \left|q\left(p_{i}+m\right)-u_{i+1}\left(p_{i}+m\right)\right| \leqslant \delta
\end{aligned}
$$

for all $i \in \mathbf{Z}$.

Proof. - The argument is classical. We repeat it here for completeness. For every $N \in \mathbf{N}$, let $q_{N}$ be a multibump solution in $\Gamma(-N, N)$; this is found by means of Theorem 4.10. Since $q_{N}$ is bounded in $L^{\infty}(\mathbf{R})$ independently of $N$, and since each $q_{N}$ solves (1), we see that $q_{N}$ is uniformly bounded in $\mathcal{C}^{2}(\mathbf{R} ; \mathbf{R})$. Therefore, by the Ascoli-Arzelà Theorem, there is a subsequence of $q_{N}$ convergent in $\mathcal{C}_{\text {loc }}^{1}(\mathbf{R} ; \mathbf{R})$ to some $q$. The fact that every $q_{N}$ solves (1) shows that $q$ solves (1) as well. Properties (29) are clearly preserved by local uniform convergence, so that $q$ is the required solution.

The previous theorem yields the existence of an uncountable number of bounded solutions to (1). If the points $p_{i}$ are chosen in a nonperiodic fashion, then these solutions will not be periodic. Therefore we obtain as a by-product the existence of an uncountable number of bounded nonperiodic orbits. On the other hand, choosing the points $p_{i}$ in a periodic way produces, with a slight modification of our arguments, infinitely many periodic solutions of (1) with arbitrarily large periods. These solutions "shadow" multibump homoclinics in each period (see [11] for a related result).

\section{COMPARISON WITH THE CLASSICAL CONDITIONS}

All the results obtained in the previous part of the paper have been proved for consecutive minimizers and under assumption $(*)$. We now wish to relate these assumptions with some classical conditions used in the geometric and perturbative approach to homoclinics. 
In order to do this we place ourselves in a setting where the classical conditions can be stated, namely we assume that the periodic minimizers $u_{0}$ and $u_{1}$ are hyperbolic. In this context (as it has been known since the time of Poincaré) the standard assumption that guarantees the existence of chaotic features in the dynamics associated to (1) is the transversality of the intersection of the stable and unstable manifolds relative to $u_{0}$ and $u_{1}$. Condition $(*)$ is strictly related to the intersection properties of these manifolds. However, as it can be understood from the autonomous case, it is only a "branch" of these manifolds that one needs to take into account; recall also that we are not assuming that $u_{1}$ is a space translate of $u_{0}$, so that we are not in a position to pass to the quotient in the phase plane. Moreover, we have always worked in the region $\left[u_{0}, u_{1}\right]$, and therefore we are only interested in what takes place in that region. For these purposes we now define stable and unstable manifolds of $u_{0}$ and $u_{1}$ relative to $\left[u_{0}, u_{1}\right]$.

Let $\phi: \mathbf{R}^{2} \rightarrow \mathbf{R}^{2}$ be the time-one map associated to Eq. (1), namely

$$
\phi(x, y)=(u(1), \dot{u}(1)) \quad \text { if and only if }\left\{\begin{array}{l}
\ddot{u}+W^{\prime}(t, u)=h(t), \\
u(0)=x, \\
\dot{u}(0)=y .
\end{array}\right.
$$

The periodic minimizers $u_{0}$ and $u_{1}$ give rise to fixed points of $\phi$.

Recall that in presence of hyperbolicity the orbits $u_{i}$ possess global stable and unstable manifolds defined, for $i=0,1$, as

$$
W^{s}\left(u_{i}\right)=\bigcup_{n \leqslant 0} \phi^{n}\left(W_{l o c}^{s}\left(u_{i}\right)\right) \quad \text { and } \quad W^{u}\left(u_{i}\right)=\bigcup_{n \geqslant 0} \phi^{n}\left(W_{l o c}^{u}\left(u_{i}\right)\right) .
$$

Roughly speaking, we now want to select from these manifolds the branches such that (denoting $P_{1}$ the projection onto the space variable), $P_{1} W^{s}\left(u_{i}\right) \subset\left[u_{0}(0), u_{1}(0)\right]$, and likewise for $W^{u}$. This corresponds, in the unforced pendulum equation, to selecting the separatrices lying in the strip $[-\pi, \pi] \times \mathbf{R}$.

To achieve this goal we first need a simple preliminary lemma. It is perhaps well known, but we prove it anyway due to the lack of a precise reference. We carry out the computations only for $u_{0}$, everything being symmetric when dealing with $u_{1}$.

Recall that $E_{n}$ is the space of $\left(H_{l o c}^{1}\right) n$-periodic functions, and set

$$
\lambda_{1}(n)=\inf _{v \in E_{n} \backslash\{0\}} \frac{\int_{0}^{n} \dot{v}^{2} d t-\int_{0}^{n} W^{\prime \prime}\left(t, u_{0}\right) v^{2} d t}{\int_{0}^{n} v^{2} d t} .
$$


Since $u_{0}$ minimizes $\int_{0}^{n} L(u) d t$ over $E_{n}$ (Lemma 2.2), we know that $\lambda_{1}(n) \geqslant 0$ for all $n$; moreover, since $u_{0}$ is now supposed to be hyperbolic, $\lambda_{1}:=\lambda_{1}(1)>0$ (a proof of this can be found in [2]).

LEMmA 5.1. - For all $n \in \mathbf{N}$ there results $\lambda_{1}(n)=\lambda_{1}$.

Proof. - Let $\varphi \in E_{n}$ be a positive eigenfunction corresponding to $\lambda_{1}(n)$. Then $\varphi$ solves

$$
-\ddot{\varphi}-W^{\prime \prime}\left(t, u_{0}\right) \varphi=\lambda_{1}(n) \varphi ;
$$

notice that, since $W^{\prime \prime}\left(\cdot, u_{0}(\cdot)\right)$ is 1-periodic, the function $\varphi(\cdot+1)$ solves (30) as well, and therefore also $z(t):=\varphi(t)-\varphi(t+1)$ is a solution. Now $z$ has zero mean value, so that it cannot solve (30) unless it is identically zero. This means that $\varphi$ is 1-periodic, and therefore $\lambda_{1}(n)$ must be an eigenvalue of the 1-periodic problem. Moreover, since $\varphi$ is positive, $\lambda_{1}(n)$ must be the first eigenvalue, namely $\lambda_{1}(n)=\lambda_{1}$.

The previous lemma provides a minimum of convexity necessary to prove a uniqueness result for solutions close to $u_{0}$.

PROPOSITION 5.2. - There exists $\sigma>0$ such that for every $\delta \in(0, \sigma)$ there exists $\varepsilon>0$ with the property that for all $x \in\left[u_{0}(0), u_{0}(0)+\varepsilon\right]$, the problem

$$
\begin{cases}\ddot{q}+W^{\prime}(t, q)=h(t) & \text { in }[0,+\infty), \\ q(0)=x, \quad q(+\infty)=u_{0} & \\ u_{0}(t) \leqslant q(t) \leqslant u_{0}(t)+\delta & \text { in }[0,+\infty),\end{cases}
$$

has a unique classical solution.

Proof. - The existence of $q$ follows with slight modifications of arguments used in the first part of the paper. Indeed it is enough to minimize the functional $J^{+}(q)=\sum_{k \geqslant 0} a_{k}(q)$ over the set

$$
\Gamma_{x}=\left\{q \in H_{l o c}^{1}\left(\mathbf{R}^{+} ; \mathbf{R}\right) \cap\left[u_{0}, u_{1}\right] / q(0)=x, q(+\infty)=u_{0}\right\} .
$$

This yields a function $q$ such that $J^{+}(q)<+\infty, q(0)=x$ and $q(+\infty) \in$ $\mathcal{U}$. In order to prove that $q(+\infty)=u_{0}$ and the remaining requirements, it is enough to show that $u_{0}(t)<q(t)<u_{0}(t)+\delta$ for all $t \geqslant 0$. But this also follows easily from arguments already used in the preceding sections, and we do not give the details of the computations.

The proof of uniqueness is very similar to the proof of Theorem 5.4 in [8]; we will therefore be rather sketchy at some points. Let $\lambda_{1}>0$ be the eigenvalue defined above, and let $\eta>0$ be such that 


$$
\left|W^{\prime \prime}(t, a)-W^{\prime \prime}(t, b)\right| \leqslant \frac{\lambda_{1}}{2}
$$

for all $t \geqslant 0$ and all $a, b$ such that $|a-b|<\eta$;

this choice is possible due to the uniform continuity of $W^{\prime \prime}$. Now if uniqueness is violated, for $\sigma=\frac{\eta}{2}$ there exist $\delta<\sigma$, a small $x$ and two solutions of (31), $q_{1}$ and $q_{2}$. Letting $\psi=q_{2}-q_{1}$, we see that $\psi$ solves

$$
\ddot{\psi}+W^{\prime}\left(t, q_{1}+\psi\right)-W^{\prime}\left(t, q_{1}\right)=0 \text { for } t \geqslant 0
$$

and satisfies $\psi(0)=0, \psi(+\infty)=0$ and $\|\psi\|_{L^{\infty}\left(\mathbf{R}^{+}\right)} \leqslant \delta$.

A standard application of the mean value Theorem (see [8]) shows that (32) can be written

$$
\ddot{\psi}+W^{\prime \prime}\left(t, u_{0}\right) \psi+a(t) \psi=0,
$$

where $|a(t)|=\left|W^{\prime \prime}\left(t, q_{1}+\theta_{t} \psi\right)-W^{\prime \prime}\left(t, u_{0}\right)\right| \leqslant \frac{\lambda_{1}}{2}$ for all $t \geqslant 0$, since $\left|q_{1}+\theta_{t} \psi-u_{0}\right| \leqslant 2 \delta<\eta$.

For every $n \in \mathbf{N}$ define now $\psi_{n}:[0, n+1] \rightarrow \mathbf{R}$ as

$$
\psi_{n}(t)= \begin{cases}\psi(t) & \text { if } t \in[0, n] \\ (n+1-t) \psi(n) & \text { if } t \in[n, n+1]\end{cases}
$$

then $\psi_{n}(n+1)=\psi_{n}(0)=0$ and therefore, by Lemma 5.1,

$$
\int_{0}^{n+1} \dot{\psi}_{n}^{2} d t-\int_{0}^{n+1} W^{\prime \prime}\left(t, u_{0}\right) \psi_{n}^{2} d t \geqslant \lambda_{1} \int_{0}^{n+1} \psi_{n}^{2} d t
$$

Recalling the definition of $\psi_{n}$, we see that the preceding inequality implies that

$$
\int_{0}^{n} \dot{\psi}^{2} d t-\int_{0}^{n} W^{\prime \prime}\left(t, u_{0}\right) \psi^{2} d t \geqslant \lambda_{1} \int_{0}^{n} \psi^{2} d t+\mathrm{o}(1) \quad \text { as } n \rightarrow \infty .
$$

On the other hand, multiplying (33) by $\psi$ and integrating over $[0, n]$ yields, as $n \rightarrow \infty$,

$$
\int_{0}^{n} \dot{\psi}^{2} d t-\int_{0}^{n} W^{\prime \prime}\left(t, u_{0}\right) \psi^{2} d t=\int_{0}^{n} a(t) \psi^{2}+\psi(n) \dot{\psi}(n)
$$




$$
\leqslant \frac{\lambda_{1}}{2} \int_{0}^{n} \psi^{2} d t+\mathrm{o}(1) .
$$

Comparing this and (34) shows that as $n \rightarrow \infty$,

$$
\frac{\lambda_{1}}{2} \int_{0}^{n} \psi^{2} d t \leqslant \mathrm{o}(1)
$$

which proves that $\psi \equiv 0$, namely that $q_{1}=q_{2}$.

We are now in a position to define stable and unstable manifolds relative to $\left[u_{0}, u_{1}\right]$.

Let $\varepsilon>0$ be given by Proposition 5.2 and, for all $x \in\left[u_{0}(0), u_{0}(0)+\right.$ $\varepsilon$ ], denote by $q_{x}$ the unique solution to (31) also given by Proposition 5.2. We define the local stable manifold of $u_{0}$ relative to $\left[u_{0}, u_{1}\right]$ as

$$
\mathcal{W}_{l o c}^{s}\left(u_{0}\right)=\left\{\left(x, \dot{q}_{x}(0)\right) \in \mathbf{R}^{2} / x \in\left[u_{0}(0), u_{0}(0)+\varepsilon\right]\right\}
$$

and the stable manifold of $u_{0}$ relative to $\left[u_{0}, u_{1}\right]$ as

$$
\mathcal{W}^{s}\left(u_{0}\right)=\bigcup_{n \leqslant 0} \phi^{n}\left(\mathcal{W}_{\text {loc }}^{s}\left(u_{0}\right)\right) \text {. }
$$

In a similar way (using obvious variants of Proposition 5.2) one can define the unstable manifold of $u_{0}$ relative to $\left[u_{0}, u_{1}\right]$ and the analogous manifolds $\mathcal{W}^{s}\left(u_{1}\right)$ and $\mathcal{W}^{u}\left(u_{1}\right)$ corresponding to $u_{1}$.

We now relate our assumptions with some classical conditions. In presence of hyperbolic periodic orbits it is well known that multibump solutions and some features of chaotic dynamics are present provided the stable and unstable manifolds of the periodic orbits intersect transversally. We show that condition $(*)$ is weaker than the transversality assumption.

THEOREM 5.3. - Let $u_{0}$ and $u_{1}$ be consecutive hyperbolic minimizers. Then

$$
\mathcal{S}_{0} \neq\left(u_{0}(0), u_{1}(0)\right) \quad \text { and } \quad \mathcal{S}_{1} \neq\left(u_{0}(0), u_{1}(0)\right)
$$

if and only if

$$
\mathcal{W}^{s}\left(u_{0}\right) \neq \mathcal{W}^{u}\left(u_{1}\right) \text { and } \mathcal{W}^{u}\left(u_{0}\right) \neq \mathcal{W}^{s}\left(u_{1}\right)
$$


Proof. - If $(*)$ holds, then by Theorem 4.10 we can find a solution $q$ asymptotic to $u_{0}$ both at $-\infty$ and at $+\infty$. Then it is readily seen that $(q(0), \dot{q}(0)) \in \mathcal{W}^{s}\left(u_{0}\right)$; however, since $q(-\infty)=u_{0}$, there also results that $(q(0), \dot{q}(0)) \notin \mathcal{W}^{u}\left(u_{1}\right)$. In the same way one proves that $(q(0), \dot{q}(0))$ belongs to $\mathcal{W}^{u}\left(u_{0}\right)$ but not to $\mathcal{W}^{s}\left(u_{1}\right)$. Working with a solution doubly asymptotic to $u_{1}$, the remaining relations can be proved similarly. Therefore $\mathcal{W}^{s}\left(u_{0}\right) \neq \mathcal{W}^{u}\left(u_{1}\right)$ and $\mathcal{W}^{u}\left(u_{0}\right) \neq \mathcal{W}^{s}\left(u_{1}\right)$ both hold.

Conversely, assume for example that there is a point $(x, y) \in \mathcal{W}^{s}\left(u_{0}\right) \backslash$ $\mathcal{W}^{u}\left(u_{1}\right)$; this means that, for a convenient $m \geqslant 0$, the point $(\bar{x}, \bar{y})=$ $\phi^{m}(x, y)$ belongs to $\mathcal{W}_{\text {loc }}^{s}\left(u_{0}\right)$. We now prove that $\bar{x} \notin \mathcal{S}_{1}$. Suppose this is not the case. Then there exists a heteroclinic minimizer $q \in \Gamma_{1}$ such that $q(0)=\bar{x}$; by choosing a larger $m$, if necessary, we can make sure that $q$ also verifies, for all $t \geqslant 0, u_{0}(t) \leqslant q(t) \leqslant u_{0}(t)+\delta$ (this is the quantity provided by Proposition 5.2). Then by uniqueness it must be $\dot{q}(0)=\bar{y}$; this means that $(\bar{x}, \bar{y})$, and therefore $(x, y)$ belongs to $\mathcal{W}^{u}\left(u_{1}\right)$, since $q(-\infty)=u_{1}$, which contradicts the choice of $(x, y)$. Therefore we must have $\bar{x} \notin \mathcal{S}_{1}$. The other case is handled in a similar way.

Remark 5.4. - Theorem 5.3 shows that working with consecutive minimizers and assuming $(*)$ is in some sense more natural than using the classical conditions. First of all in presence of hyperbolicity $(*)$ is weaker than the usual transversality assumption. Moreover $(*)$ can be used, like we did, also without hyperbolicity, when the classical condition cannot be stated. Finally we observe that the variational characterization of the numbers $c_{0}$ and $c_{1}$ which is used to introduce $(*)$ shows that the validity of that condition is stable under small perturbations. This allows one to say, for example, that the set of $h$ 's for which $(*)$ holds is open in the space of continuous, zero mean valued periodic functions.

Remark 5.5. - As a final remark we observe that a family of multibump solutions like those provided by Theorem 4.10 ensures the positivity of the topological entropy for the map $\phi$. We do not prove this statement because its proof is essentially the same as those in [20] and [7].

\section{ACKNOWLEDGEMENT}

The authors wish to thank their friend Susanna Terracini for some stimulating and useful discussions. 


\section{REFERENCES}

[1] Alessio F., Calanchi M., Serra E., Complex dynamics in a class of reversible equations, in: Proc. of Autumn School on Nonlinear Analysis and Differential Equations, Lisbon, 1998, to appear.

[2] Amann H., Ordinary Differential Equations, De Gruyter, Berlin, 1990.

[3] Ambrosetti A., Badiale M., Homoclinics: Poincaré-Melnikov type results via a variational approach, Ann. IHP, Anal. non Lin. 15 (1998) 233-252.

[4] Bangert V., Mather sets for twist maps and geodesics on tori, in: Dinamics Reported, Vol.1, Teubner, 1988, pp. 1-56.

[5] Bolotin S.V., The existence of homoclinic motions, Vest. Mosk. Univ., Matem. 38 (1983) 98-103.

[6] Bolotin S.V., Rabinowitz P.H., A variational construction of chaotic trajectories for a Hamiltonian system on a torus, Boll. UMI 1 (1998) 541-570.

[7] Buffoni B., Séré E., A global condition for quasi-random behavior in a class of conservative systems, Comm. Pure Appl. Math. 49 (1996) 285-305.

[8] Calanchi M., Serra E., Homoclinic solutions to periodic motions in a class of reversible equations, Calc. Var. and PDEs. 9 (1999) 157-184.

[9] Coti Zelati V., Ekeland I., Séré E., A variational approach to homoclinic orbits in Hamiltonian systems, Math. Annalen 288 (1990) 133-160.

[10] Coti Zelati V., Rabinowitz P.H., Homoclinic orbits for second order Hamiltonian systems possessing superquadratic potentials, J. AMS 4 (1991) 693-727.

[11] Coti Zelati V., Rabinowitz P.H., Multibump periodic solutions for a family of Hamiltonian systems, Topol. Methods in Nonlinear Anal. 4 (1995) 31-57.

[12] Mather J.N., Variational construction of connecting orbits, Ann. Inst. Fourier 43 (1993) 1349-1386.

[13] Maxwell T.O., Heteroclinic chains for a reversible Hamiltonian system, Nonlin. Anal. TMA 28 (1997) 871-887.

[14] Montecchiari P., Nolasco M., Terracini S., A global condition for periodic Duffinglike equations, Trans. AMS 351 (1999) 3713-3724.

[15] Offin D.C., Yu H.-F., Homoclinic orbits in the forced pendulum system, Fields Inst. Comm. 8 (1996) 113-126.

[16] Rabinowitz P.H., Heteroclinics for a reversible Hamiltonian system, Ergodic Theory Dynamical Systems 14 (1994) 817-829.

[17] Rabinowitz P.H., Heteroclinics for a reversible Hamiltonian system, 2, Differential Integral Equations 7 (1994) 1557-1572.

[18] Rabinowitz P.H., Connecting orbits for a reversible Hamiltonian system, Ergodic Theory Dynamical Systems, to appear.

[19] Séré E., Existence of infinitely many homoclinic orbits in Hamiltonian systems, Math. Zeit. 209 (1992) 27-42.

[20] Séré E., Looking for the Bernoulli shift, Ann. IHP, Anal. non Lin. 10 (1993) 561590.

[21] Serra E., Tarallo M., Terracini S., On the structure of the solution set of forced pendulum-type equations, J. Differential Equations 131 (1996) 189-208.

[22] Terracini S., Nondegeneracy and chaotic motions for a class of almost-periodic Lagrangian systems, Nonlin. Anal. TMA 37 (1999) 337-361.

[23] Wiggins S., Introduction to Applied Nonlinear Dynamical Systems and Chaos, Springer Verlag, New York, 1990. 